\title{
On Index Theorem for Symplectic Orbifolds
}

\author{
B. Fedosov B.-W. Schulze N. Tarkhanov \\ Universität Potsdam, Institut für Mathematik, Postfach 6015 53, 14415 Potsdam, Germany
}

June 27, 2003

\begin{abstract}
We give an explicit construction of the trace on the algebra of quantum observables on a symplectic orbifold and propose an index formula.
\end{abstract}

2000 Mathematics Subject Classifications: primary: 53D55; secondary: 37J10.

Key words: star product, symmetry group, $G$-trace, $G$-index.

\section{Introduction}

This paper was originally planned as a field for cooperation between analysis of partial differential operators on manifolds with singularities on the one hand, and deformation quantization of symplectic manifolds on the other hand. A symplectic orbifold seems to be a good object of interest for the following reasons:

$1^{\circ}$ Orbifolds may have rather complicated singularities but with a rich symmetry. In particular, the symmetry conditions of [17], [9] leading to essential simplifications of the index theory are a part of the orbifold structure. The index theory for elliptic operators on compact orbifolds takes its final form due to the works of Kawasaki [13] and Vergne [18]. Their formulas show that for orbifolds we have well-defined contributions of the smooth part and of singularities.

$2^{\circ}$ Deformation quantization as described in [4] goes almost without changes for the symplectic orbifolds, cf. [14].

Our hope was that combining all these methods, we could generalize the index theorem for deformation quantization on smooth symplectic manifolds to the case of symplectic orbifolds. Unfortunately, we could not realize our plans in their original form. Instead, some new interesting observations concerning different versions of quantization, traces, eigenvalue problems were made in 
the process. These observations generate more questions than answers, and we hope to continue somewhere our investigations in these directions.

Our main result is the trace construction on the algebra of quantum observables on a symplectic orbifold. This is done in Section 7 using the local study of orbifold charts (Section 2) and orbifold vector bundles (Section 3). In contrast to the smooth case the orbifold trace is not unique. On the set of traces acts a finite group which we call the Picard group. Its description as global sections of the sheaf of characters is given in Section 4. It seems plausible that the trace is unique up to the action of the Picard group but at the moment we can not prove this statement.

Section 5 contains a standard material on symplectic reduction. In particular, we show that any symplectic orbifold may be obtained by symplectic reduction of a smooth symplectic manifold by a locally free Hamiltonian action of a compact connected Lie group. It is a necessary step in the index theory of Kawasaki and Vergne which reduces the orbifold index theorem to the Atiyah index theorem for transversally elliptic operators [1]. We use the symplectic reduction when considering examples in Section 9.

In Section 6 a brief description of the standard deformation quantization procedure on symplectic orbifolds is given. We follow here [5] paying attention to the ideas rather than the rigorous exposition.

Having constructed the trace, we define the index as the trace of the identity element in the algebra of quantum observables. This is the simplest version valid for compact symplectic orbifolds. We express the index as the sum of contributions of fixed point orbifolds, but the form of each contribution remains a conjecture which we can not prove at the moment. The matter is that the generalization of the Atiyah theory of transversally elliptic operators is missing for deformation quantization and we need either to develop it from the very beginning or to invent some new tools. We hope to prove this conjecture in future.

In the last Section 9 we try to explain that there is a reasonable version of an eigenvalue problem in the framework of deformation quantization based on the index theorem. The index is interpreted as a multiplicity of an eigenvalue and the characteristic equation for eigenvalues appears by equating this multiplicity to a positive integer number. The example also illustrates an essential role of the Picard group in such an eigenvalue problem.

To our mind, our results, although incomplete, shed some new light on the whole problem of quantization. There is a variety of quantization theories, and this very fact shows that the right quantization theory is not yet invented. Let us mention some of them. First of all it is the deformation quantization [2] which during the latest years became a "fashionable" theory. The Planck constant $h$ is treated in deformation quantization as a formal parameter and the observables are of the same nature as in the classical mechanics with quantum 
corrections given by higher powers of a formal power series in $h$. This approach gives the maximal freedom, when dealing with observables, because almost any classical construction with observables admits a lifting to the quantum case. On the other hand, the quantum evolution defined by the Schrödinger operator $\exp ((\imath / h) H t)$ is lost in the deformation quantization approach. Moreover, the spectral theory is also lost since the spectrum is given by the Fourier expansion of the evolution operator.

Another quantization theory coming from the group representations is the so-called geometric quantization. Here $h$ is a number, the quantum observables are operators, so the spectrum is correctly defined. The price for that is a complicated nature of observables, so that the lifting of classical constructions to the quantum level becomes non-trivial.

We also mention the Maslov quantization theory based on a version of Fourier integral operators, cf. [11]. It tries to combine the merits of both above-mentioned approaches, but possesses the shortcomings of the both as well.

Our approach to quantization is the following. If one wishes to describe the global picture, one needs to deal within the scope of deformation quantization. But when considering the local details one may use the operator representation of local deformation quantum algebra in case it exists. So, in the local picture one takes $h$ as a small numerical parameter, and to compute the quantity in question one uses the operator machinery. If the result admits an asymptotic expansion in powers of $h$ as $h$ tends to 0 , one may treat this asymptotic series as a formal one and thus return to the deformation setting. The only thing one needs to check is that the final expression treated as a formal series is independent of the operator representation which has been used for calculations. For example, the index formula usually possesses such invariance property because of the topological stability. Such reasoning was developed in the proof of the index theorem for deformation quantization in [5]. Here we also use this reasoning, when constructing the traces for quantum algebras on symplectic orbifolds, see also the discussion in Section 7.

\section{Symplectic orbifolds}

As a background on orbifolds we recommend the book [3].

A symplectic orbifold is a Hausdorff topological space $B$ which admits a locally finite covering $\left\{O_{i}\right\}$ with the following properties:

$1^{o}$ For each $O_{i}$ there exists a domain $\widetilde{O}_{i}$ in the standard symplectic space $\left(\mathbb{R}^{2 n}, \omega\right)$ and a finite group $G_{i}$ of symplectomorphisms of $\widetilde{O}_{i}$, such that $O_{i}$ is homeomorphic to the orbit space $\widetilde{O}_{i} / G_{i}$. We denote by $p_{i}$ the corresponding projection $p_{i}: \widetilde{O}_{i} \rightarrow \widetilde{O}_{i} / G_{i}=O_{i}$. The domains $\widetilde{O}_{i}$ with the given group action 
are called orbifold charts.

$2^{o}$ If an open set $O$ belongs to the intersection $O_{j} \cap O_{k}$, then there exists a domain $\widetilde{O}$ in $\left(\mathbb{R}^{2 n}, \omega\right)$ with a finite group $G$ of symplectomorphisms of $\widetilde{O}$ and a projection $p: \widetilde{O} \rightarrow \widetilde{O} / G=O$ subject to the condition: there are symplectomorphisms

$$
\widetilde{O}_{j} \stackrel{\varphi_{j}}{\longleftarrow} \widetilde{O} \stackrel{\varphi_{k}}{\longrightarrow} \widetilde{O}_{k}
$$

and group embeddings

$$
G_{j} \stackrel{i_{j}}{\leftarrow} G \stackrel{i_{k}}{\rightarrow} G_{k}
$$

such that the maps (2.1) are equivariant with respect to the homomorphisms $(2.2)$.

For a point $x \in O_{i}$ take one of its preimages $\tilde{x} \in \widetilde{O}_{i}$ and consider a subgroup $G(\tilde{x}) \subset G_{i}$ leaving $\tilde{x}$ fixed. It is called a stabilizer or isotropy subgroup of $\tilde{x}$. Any other preimage has the form $\gamma \tilde{x}$ for some $\gamma \in G_{i}$, so that

$$
G(\gamma \tilde{x})=\gamma G(\tilde{x}) \gamma^{-1}
$$

We see that $G(\gamma \tilde{x})$ may be obtained by conjugation from $G(\tilde{x})$. In particular, $G(\gamma \tilde{x})$ and $G(\tilde{x})$ are isomorphic, and we may introduce the stabilizer of $x \in O_{i}$ as a group isomorphic to any of $G(\tilde{x})$. Moreover, the conditions (2.1), (2.2) imply that $G(x)$ is independent of the chart up to an isomorphism, so the notion of the isotropy group $G(x)$ makes sense for a point $x \in B$.

Theorem 2.1 For any compact symplectic orbifold $B$ there exists a covering $\left\{O_{j}, \widetilde{O}_{j}, G_{j}, p_{j}\right\}$ by orbifold charts with the following properties:

$1^{o}$ In each $O_{j}$ there exists a point $x_{j}$ called the center, such that $G\left(x_{j}\right)$ coincides with $G_{j}$.

$2^{o}$ There is a $G_{j}$-invariant complex structure on $\widetilde{O}_{j}$, such that $\widetilde{O}_{j}$ is a neighbourhood of the origin in $\mathbb{C}^{n}$ with the symplectic form

$$
\omega=\frac{1}{2 \imath}\left(d \bar{z}_{1} \wedge d z_{1}+\ldots+d \bar{z}_{n} \wedge d z_{n}\right)
$$

$3^{o} G_{j}$ acts on $\widetilde{O}_{j}$ by unitary transformations, that is $G_{j}$ is a finite subgroup of $\mathrm{U}(n)$.

This is the so-called linearization theorem.

Proof. Take a point $x_{0} \in B$ and an orbifold chart $\{O, \widetilde{O}, G, p\}$ containing $x_{0}$. Pick one of the preimages $\tilde{x}_{0} \in \widetilde{O}$ and a smaller neighbourhood $\widetilde{O}_{1} \subset \widetilde{O}$, such that $\gamma \widetilde{O}_{1} \cap \widetilde{O}_{1} \neq \emptyset$ implies $\gamma \in G\left(\tilde{x}_{0}\right)$. Then $\left\{p\left(\widetilde{O}_{1}\right)=O_{1}, \widetilde{O}_{1}, G\left(\tilde{x}_{0}\right), p\right\}$ is a smaller orbifold chart. Because of compactness we may choose a finite covering of such form, proving $1^{\circ}$. Further we will consider only orbifold charts together with their centers. 
Let $\{O, \widetilde{O}, G, p\}$ be an orbifold chart with the center $x_{0}$, so that $\tilde{x}_{0}$ coincides with $0 \in \mathbb{R}^{2 n}$. Introduce a $G$-invariant Riemannian metric $\alpha$ on $\tilde{O}$ (averaging over a finite group $G$ makes any metric $G$-invariant). On the tangent space $T_{0} \mathbb{R}^{2 n}=\mathbb{R}^{2 n}$ the group $G$ acts by orthogonal linear transformations. The exponential map defined by $\alpha$

$$
\exp _{0}: T_{0} \mathbb{R}^{2 n} \rightarrow \mathbb{R}^{2 n}
$$

intertwines this linear action with the original action on $\widetilde{O} \subset \mathbb{R}^{2 n}$. Thus, in normal coordinates the inclusion $G \subset \mathrm{SO}(2 n)$ is valid, in other words, we have linearized the group action. The determinant is equal to 1 because the orientation given by the form $\omega$ is preserved.

Consider now the form $\omega$ in normal coordinates

$$
\omega=\frac{1}{2} \omega_{i j}(x) d x^{i} \wedge d x^{j}
$$

We apply a $G$-invariant version of the Weinstein-Moser trick to reduce $\omega$ to the constant form

$$
\omega_{0}=\frac{1}{2} \omega_{i j}(0) d x^{i} \wedge d x^{j} .
$$

Consider the family of forms $\omega(t)=\omega_{0}+t\left(\omega-\omega_{0}\right)$. For each $t \in[0,1]$ they are $G$-invariant and

$$
\omega-\omega_{0}=d \lambda,
$$

where $\lambda=\lambda_{i}(x) d x^{i}$ is a one-form. We may assume that $\lambda$ is $G$-invariant since the averaging defines a new form $\lambda$ also satisfying (2.5). Moreover, we may assume that $\lambda_{i}(0)=0$, otherwise we replace it by $\left(\lambda_{i}(x)-\lambda_{i}(0)\right) d x^{i}$, which also satisfies $(2.5)$ and is $G$-invariant. It gives a $G$-invariant vector field $X_{t}$ vanishing at the origin and satisfying

$$
i\left(X_{t}\right) \omega(t)=-\lambda \text {. }
$$

The flow $f_{t}(x)$ of this vector field is defined on the whole interval $t \in[0,1]$ for sufficiently small $x$, because $X_{t}(0)=0$. Moreover, the flow commutes with the linear $G$-action since $X_{t}$ is $G$-invariant. Then $f_{1}^{*} \omega=\omega_{0}$, because, in virtue of the Cartan homotopy formula,

$$
\begin{aligned}
\frac{d}{d t} f_{t}^{*} \omega(t) & =f_{t}^{*}\left(\frac{\partial}{\partial t} \omega(t)+\mathcal{L}_{X_{t}} \omega(t)\right) \\
& =f_{t}^{*}\left(\omega-\omega_{0}+\operatorname{di}\left(X_{t}\right) \omega(t)\right) \\
& =f_{t}^{*}(d \lambda-d \lambda) \\
& =0 .
\end{aligned}
$$


At this step we have a constant symplectic form $\omega_{0}$ preserved by the group $G \subset \mathrm{SO}(2 n)$.

Taking any constant $G$-invariant metric $\alpha$ and applying a standard construction (see for instance [5, Ch. 2]), we obtain a $G$-invariant positive complex structure $J$ and a new metric $\alpha_{0}=\omega_{0}(J \cdot, \cdot)$. The symplectic space $\mathbb{R}^{2 n}$ with the complex structure $J$ becomes $\mathbb{C}^{n}$, the form $\alpha_{0}+\imath \omega_{0}$ gives an Hermitian metric and the group $G$ which preserves this Hermitian metric is thus a subgroup of $\mathrm{U}(n)$.

For paracompact orbifolds the existence of such a linearized covering will be assumed. In some cases (for example, for vector bundles over a compact base $B$ ) this assumption may be proved.

Next, we consider the whole set of symplectic linearizations. We will show that this set is connected: any two linearizations may be linked by a smooth one-parameter family. This fact will be crucial for the trace construction in Section 7 .

Let $\widetilde{O}$ be an orbifold chart with the finite group $G$ of symplectomorphisms. Suppose we have two Darboux coordinate systems in $\widetilde{O}$, namely $x=\left(x^{1}, \ldots, x^{2 n}\right)$ and $y=\left(y^{1}, \ldots, y^{2 n}\right)$, so that $G$ acts by linear symplectomorphisms in both coordinate systems

$$
g: \begin{aligned}
x & \mapsto g x, \\
y & \mapsto \tilde{g} y,
\end{aligned}
$$

where $g, \tilde{g} \in \operatorname{Sp}(2 n)$. To put it differently, $x$ and $y$ are related by a non-linear symplectomorphism

$$
y=f(x)
$$

with the property

$$
f(g x)=\tilde{g} f(x), \quad g \in G .
$$

Theorem 2.2 There exists a smooth family $f_{t}(x), t \in[0,1]$, of symplectomorphisms, such that $f_{0}(x)=x, f_{1}(x)=f(x)$ and $f_{t}(x)$ satisfies the relation (2.9) for each $t \in[0,1]$.

Proof. Consider first the case when the linear part of (2.8) is identity, that is

$$
f(x)=x+\varphi(x)=x+O\left(|x|^{2}\right) .
$$

Comparing the linear parts on both sides of (2.10), we see that $g=\tilde{g}$. To construct the homotopy $f_{t}(x)$, we will use generating functions (see for instance [5, Section 2.4] or [6]).

Let the symplectomorphism (2.8) correspond to the Cayley generating function $S$, that is, the relation $y=f(x)$ is obtained by elimination of the auxiliary 
variable $z$ from the following two equations

$$
\begin{aligned}
& x=z-\nabla S(z) \\
& y=z+\nabla S(z) .
\end{aligned}
$$

Here $\nabla S$ means the symplectic gradient, it is a vector satisfying the relation $i(\nabla S) \omega=-d S$, or in coordinates

$$
\nabla^{i} S=\omega^{i j} \frac{\partial S}{\partial z^{j}}
$$

From (2.11) it follows that

$$
\begin{aligned}
z & =\frac{x+y}{2}=\frac{x+f(x)}{2}, \\
\nabla S & =\frac{x-y}{2}
\end{aligned}
$$

and further, taking $S(0)=0$, we get

$$
S(z)=\int_{0}^{z} i\left(\frac{x-y}{2}\right) \omega .
$$

It is easy to verify that equations (2.11) actually define a symplectomorphism, provided we can express $z$ as an implicit function of $x$ from the first equation (2.11). This is always the case in a neighborhood of the origin if $S(z)$ has a third order zero at $z=0$. Vice versa, if the linear part of $f(x)$ is identity, then $x$ may be defined as an implicit function of $z$ and the integrand in(2.13) is a closed form.

Lemma 2.3 A symplectomorphism $y=f(x)$ defined by a generating function $S(z)$ satisfies (2.9) if and only if the generating function is invariant, i.e.

$$
S(g z)=S(z), \quad g \in G .
$$

Proof. Differentiating (2.14), we get

$$
\nabla^{i} S(z)=\omega^{i j} g_{j}^{k} \frac{\partial S}{\partial z^{k}}(g z)
$$

and further, applying $g$ to both sides,

$$
\begin{aligned}
g_{i}^{l} \nabla^{i} S(z) & =g_{i}^{l} \omega^{i j} g_{j}^{k} \frac{\partial S}{\partial z^{k}}(g z) \\
& =\omega^{l k} \frac{\partial S}{\partial z^{k}}(g z) .
\end{aligned}
$$


Here we have used the fact that $g$ is a symplectic matrix, thus $g_{i}^{l} \omega^{i j} g_{j}^{k}=\omega^{l k}$. In other words, the vector $\nabla S(z)$ satisfies the relation

$$
g(\nabla S(z))=\nabla S(g z)
$$

Then (2.11) yields

$$
\begin{aligned}
& g x=g z-\nabla S(g z)=\widetilde{z}-\nabla S(\widetilde{z}) \\
& g y=g z+\nabla S(g z)=\widetilde{z}+\nabla S(\widetilde{z}),
\end{aligned}
$$

where we have denoted $g z$ by $\widetilde{z}$. Eliminating $\widetilde{z}$ from these equations, we see that $g x$ and $g y$ satisfy the same relation $g y=f(g x)$ as $x$ and $y$, whence

$$
g f(x)=f(g x) .
$$

Vice versa, let $(2.9)$ be fulfilled. Then the action $x \mapsto g x$ implies

$$
\begin{aligned}
y & =f(x) \mapsto g y, \\
z & =\frac{x+y}{2} \mapsto g z, \\
\nabla S & =\frac{x-y}{2} \mapsto g(\nabla S) .
\end{aligned}
$$

We thus get

$$
\nabla S(g z)=g(\nabla S(z))
$$

and this, in turn, implies in virtue of $(2.14)$ that $S(z)$ is invariant

Now, to construct the homotopy $f_{t}(z)$, we take the generating function $S(z)$ of the original symplectomorphism $f(x)$, multiply it by $t \in[0,1]$ and then consider the symplectomorphisms defined by the generating functions $t S(z)$. Because of (2.15) $S(z)$ is invariant, hence so is $t S(z)$, implying that (2.15) is fulfilled for any $t$. This proves the theorem in the special case (2.10).

In the general case we rewrite the symplectomorphism $f(x)$ in the form

$$
y=a(b+x+\varphi(x))
$$

where $a b+a x$ is a linear part and $\varphi(x)=O\left(|x|^{2}\right)$. Thus, $a=f^{\prime}(0), a b=f(0)$, and the property (2.9) gives

$$
\tilde{g} a(b+x+\varphi(x))=a(b+g x+\varphi(g x)) .
$$

Hence it follows that $\tilde{g}=a g a^{-1}, g b=b$ and $\varphi(g x)=g \varphi(x)$. The group $\operatorname{Sp}(2 n)$ is connected, thus there exists a path $a_{t} \in \operatorname{Sp}(2 n)$ linking $a$ with the identity matrix 1. After this homotopy the symplectomorphism (2.16) takes the form $y=b+x+\varphi(x)$ and, moreover, $\tilde{g}=g$. The vector $b$ belongs to $\operatorname{ker}(g-1)$ for 
any $g \in G$, and so does $t b$ for any $t \in[0,1]$. Thus, we can pull $b$ to zero. Our symplectomorphism then becomes

$$
y=x+\varphi(x),
$$

and this can be linked to the identity, as was already proved.

Remark 2.4 The homotopy $f_{t}(x)$ constructed in the theorem may be extended to a positive complex structure $J$. Indeed, let $J$ be a constant complex structure making the symplectic space $\mathbb{R}^{2 n}$ into $\mathbb{C}^{n}$ and the group $G \in \operatorname{Sp}(2 n)$ into a subgroup of $\mathrm{U}(n)$. It defines a constant metric $\alpha=\omega(J \cdot, \cdot)$. Having a homotopy $y=f_{t}(x)$ of the constant symplectic structure and the corresponding group $G_{t} \in \operatorname{Sp}(2 n)$, we define a new metric $\alpha_{t}$ by averaging $\alpha$ with respect to $G_{t}$ and then construct a positive complex structure $J_{t}$ in a standard way starting with two bilinear forms $\omega$ and $\alpha_{t}$.

The group $G$ for an orbifold chart $\widetilde{O}$ is not uniquely defined. For example, the chart $O=\mathbb{C} / \mathbb{Z}_{3}$, where $\mathbb{Z}_{3}$ acts by multiplication by

$$
\exp \left(2 \pi \imath \frac{k}{3}\right), \quad k \in \mathbb{Z} \bmod 3,
$$

may be replaced by the chart $\mathbb{C} / \mathbb{Z}_{6}$ with the same action as in (2.17) but with $k \in \mathbb{Z} \bmod 6$. In the second case the action is not effective: the subgroup with $k=3 l \bmod 6$ acts as identity. In general, denoting by $G_{0} \subset G$ a subgroup which acts on $\widetilde{O}$ as identity, we can pass on to the effective action by replacing $G$ by the quotient $G / G_{0}$ (clearly, $G_{0}$ is a normal subgroup). So, we will assume as a rule that the actions of $G_{i}$ on $\widetilde{O}_{i}$ are effective. In this case we have an open dense set $B_{0} \subset B$, the so-called principal stratum, such that each point $x \in B_{0}$ has a trivial stabilizer. This is a smooth part of the orbifold $B$. The remaining points form singularities, which in general may be very complicated. In the above example (2.17) we have the only singular point $z=0$ which is a conical point.

Singular points (the points with non-trivial stabilizer) admit further stratification but we will not touch this subject here.

Although $B$ may have rather complicated singularities, the notion of a smooth function still makes sense for orbifolds. Namely, $f \in C^{\infty}(B)$ if its lifting to any orbifold chart $\widetilde{O}$ is smooth and necessarily $G$-invariant, that is

$$
f(\gamma \tilde{x})=f(\tilde{x}) .
$$

Thus, a possibility appears to develop analysis and differential geometry on orbifolds. 


\section{Orbifold vector bundles}

The notion of orbifold vector bundle requires some precautions. A naive definition is that $E$ is a continuous vector bundle over a topological space $B$, which may be described by means of smooth functions. For example, any matrix-valued function

$$
P(x)=\left(p_{i j}(x)\right)
$$

whose values are projectors and whose entries $p_{i j}(x)$ are smooth functions defines an orbifold vector bundle. But this definition is too restrictive, even tangent and cotangent bundles do not fit into this scheme.

Definition 3.1 An orbifold vector bundle $E$ is an object which in an orbifold chart $\{O, \widetilde{O}, G, p\}$ is given by a $G$-equivariant vector bundle $\widetilde{E}_{G}$. These local equivariant bundles should be compatible with respect to symplectomorphisms (2.1) and homomorphisms (2.2).

Thus, the total space of $E$ is an orbifold itself, with the same local groups $G_{i}$ as for the base $B$. Sometimes one considers a more general case when the local groups of $E$ are central extensions of $G_{i}$, in this case the bundle is called improper orbifold vector bundle.

For the time being we will deal with proper bundles. In this case we have a linearization theorem similar to Theorem 2.1.

Theorem 3.2 Let $E$ be a compact proper orbifold vector bundle. Then for any linearized orbifold chart $\{O, \widetilde{O}, G, p\}, G \subset \mathrm{U}(n)$, there exists a frame of the bundle $\widetilde{E}_{G}$ and a complex representation $g \mapsto T(g) \in \operatorname{End}\left(E_{0}\right)$, such that the sections $s(z, \bar{z})$ are vector-valued functions with values in $E_{0}$, and the group $G$ acts on sections as follows

$$
s(g z, \overline{g z})=T(g) s(z, \bar{z}) .
$$

Proof. The point $\tilde{x} \in \widetilde{O}$ may be written as a pair $z, \bar{z}$ with $z \in \mathbb{C}^{n}$, and we will use both designations $\tilde{x}$ and $z, \bar{z}$. Choosing any frame of $\widetilde{E}_{G}$ over $\widetilde{O}$, we consider the sections $s(\tilde{x})$ as vector-valued functions. By the definition of the equivariant vector bundle we have the action of the group $G$ on sections by $s(g \tilde{x})=T(g)(\tilde{x}) s(\tilde{x})$, where the matrix-valued function $T(g)(\tilde{x})$ defines a linear map

$$
T(g)(\tilde{x}): \widetilde{E}_{\tilde{x}} \rightarrow \widetilde{E}_{g \tilde{x}} .
$$

At the origin $0 \in \widetilde{O}$ which is a fixed point we have thus endomorphisms $T(g)(0): \widetilde{E}_{0} \rightarrow \widetilde{E}_{0}$, and this is the desired representation. Clearly, we can make it unitary by introducing a Hermitian metric and averaging it over the finite group. 
In order to construct the needed frame, we now choose a $G$-invariant Hermitian connection on $E$ (again using the averaging), take any unitary frame in $\widetilde{E}_{0}$ and spread it over the whole $\widetilde{O}$ by parallel transports along the rays $t \tilde{x}$, $t \in[0,1]$.

Remark 3.3 For linearized coordinates $z, \bar{z}$ and frames (3.2), the simplest invariant connection is given by the de Rham differential $d s(z, \bar{z})$ of vectorvalued functions.

Now, smooth vector fields, differential forms and other geometric objects are defined as sections of corresponding orbifold vector bundles $T B, T^{*} B$ and so on.

The integral of a differential form of the top degree over the orbifold $B$ is defined in an obvious way. We take a smooth partition of unity $\rho_{i}(x)$ on $B$. When lifted to an orbifold chart $\widetilde{O}_{i}$, the function $\rho_{i}(\tilde{x})$ is smooth and $G_{i}$-invariant, and we define

$$
\int_{B} \alpha=\sum_{i} \frac{1}{\left|G_{i}\right|} \int_{\widetilde{O}_{i}} \rho_{i}(\tilde{x}) \alpha_{i}
$$

where $\alpha_{i}$ is a local expression of the form $\alpha$. Now, since $\gamma^{*}\left(\rho_{i} \alpha_{i}\right)=\chi_{i}(\gamma) \rho_{i} \alpha_{i}$ where $\chi_{i}(\gamma)$ is a one-dimensional character, the integrals are equal to zero unless $\chi_{i}(\gamma) \equiv 1$. In the latter case we may consider $\rho_{i}(\tilde{x}) \alpha_{i}$ as the form coming from $B$, and the integral will be simply equal to

$$
\int_{B_{0}} \alpha
$$

that is the integral over the smooth part $B_{0}$ of $B$.

We have tacitly assumed that the group $G_{i}$ acts effectively. In general, when the action is not effective, we pass on to the quotient $\widetilde{G}_{i}=G_{i} / G_{0}$ where $G_{0}$ is a normal subgroup of $G_{i}$ acting as identity. By compatibility conditions (2.1) and (2.2), the number $m(B)=\left|G_{0}\right|$ does not depend on the chart (for connected orbifolds), it is called the multiplicity of the orbifold. Then one defines

$$
\begin{aligned}
\int_{B} \alpha & =\sum_{i} \frac{1}{\left|\widetilde{G}_{i}\right|} \int_{\widetilde{O}_{i}} \rho_{i}(\tilde{x}) \alpha_{i} \\
& =m(B) \sum_{i} \frac{1}{\left|G_{i}\right|} \int_{\widetilde{O}_{i}} \rho_{i}(\tilde{x}) \alpha_{i}
\end{aligned}
$$

\section{The Picard group}

Consider more attentively the case of one-dimensional orbifold vector bundles. The representations $T(g)$ of Theorem 3.2 in linearized orbifold charts are onedimensional characters $\chi(g)$, that is homomorphisms of the group $G$ into $\mathrm{U}(1)$. 
The values are roots of unity since the group $G$ is finite. Thus, the values of $\chi(g)$ have the form

$$
\exp \left(2 \pi \imath \frac{k}{n}\right)
$$

with $k \in \mathbb{Z} \bmod n$.

A still more particular case we obtain if the representation $T(g)$ is the restriction of a representation of the whole unitary group $\mathrm{U}(n)$ to the subgroup $G$. Such one-dimensional representation has the form $\chi(g)=(\operatorname{det} g)^{k}$ with $k \in \mathbb{Z}$. Sometimes one considers multi-valued characters, where $k$ is rational, that is a representation of the finite covering of $\mathrm{U}(n)$. This leads to improper one-dimensional bundles.

Clearly, one-dimensional bundles form a commutative group with respect to tensor product. Moreover, this group acts on any vector bundle by tensoring. Under tensoring the local representations are multiplied, so that $T(g)$ goes to $\chi(g) T(g)$. Thus, we have a subgroup of one-dimensional bundles with trivial local characters. In other words, these bundles are defined over a space $B$ and have a smooth description as the orbifold bundles. We denote the group of all one-dimensional orbifold bundles by $E^{1}$ and the subgroup by $E_{B}^{1}$.

Definition 4.1 By the Picard group is meant the quotient

$$
\operatorname{Pic}=E^{1} / E_{B}^{1}
$$

The notion of Picard group is specific for orbifolds and will play essential role in the sequel, so, we need to consider it in more detail. Roughly speaking, the elements of the Picard group are trivial (flat) one-dimensional bundles with non-trivial local group actions. More precisely, they are global sections of the sheaf of characters as described below.

Given a group $G$, we denote by $\chi(G)$ the set of all homomorphisms of $G$ to $\mathrm{U}(1) \cong \mathbb{S}^{1}$, i.e., $\chi\left(g_{1} g_{2}\right)=\chi\left(g_{1}\right) \chi\left(g_{2}\right)$ fulfills for all $g_{1}, g_{2} \in G$. The product of two characters is a character, hence $\chi(G)$ is a group. By the direct product $G \times H$ of two groups $G$ and $H$ is meant the set $\{(g, h): g \in G, h \in H\}$ along with the operation $\left(g_{1}, h_{1}\right)\left(g_{2}, h_{2}\right):=\left(g_{1} g_{2}, h_{1} h_{2}\right)$. It is easy to see that $G \times H$ is a group, the unit of this group being $(1,1)$. One can specify $G, H$ within the product $G \times H$ by identifications $G \ni g \mapsto(g, 1) \in G \times H$ and $H \ni h \mapsto(1, h) \in G \times H$. If $\chi$ is a character on the direct product $G \times H$, then

$$
\begin{aligned}
\chi(g, h) & =\chi((g, 1)(1, h)) \\
& =\chi(g, 1) \chi(1, h) \\
& =\chi_{G}(g) \chi_{H}(h)
\end{aligned}
$$

where $\chi_{G}(g)=\chi(g, 1)$ and $\chi_{H}(h)=\chi(1, h)$ are characters on $G$ and $H$, respectively, determined by $\chi$ in a unique way. Conversely, given any characters 
$\chi_{G}$ and $\chi_{H}$ on $G$ and $H$, respectively, the function $\chi(g, h):=\chi_{G}(g) \chi_{H}(h)$ is a character on $G \times H$.

In the same manner we define the direct product of a finite number of groups $\prod_{j} G_{j}$. The $j$-th factor $G_{j}$ can be specified within the product by the identification $G_{j} \ni g_{j} \mapsto\left(1, \ldots, 1, g_{j}, 1, \ldots, 1\right) \in \prod_{j} G_{j}$, where $g_{j}$ takes the $j$-th position. Any character on $\prod_{j} G_{j}$ is then of the form $\chi(g)=\prod_{j} \chi_{j}\left(g_{j}\right)$ where $\chi_{j}$ is a character on the group $G_{j}$, uniquely determined by $\chi$, and conversely. Write $\chi\left(\prod_{j} G_{j}\right)$ for the set of all characters on the product. Once again $\chi\left(\prod_{j} G_{j}\right)$ is a group.

We are now in a position to introduce the sheaf of characters $\mathcal{S}$ over the base $B$. Let $O$ be an arbitrary open set in $B$. By assumption there are a finite number of charts $\left\{O_{j}, \widetilde{O}_{j}, G_{j}, p_{j}\right\}$, such that $O \subset \cup_{j} O_{j}$. If $O_{j} \cap O_{k} \neq \emptyset$ then there exists a domain $\widetilde{O}_{j k}$ in $\left(\mathbb{R}^{2 n}, \omega\right)$ with a finite group $G_{j k}$ of symplectomorphisms, and a projection $p_{j k}: \widetilde{O}_{j k} \rightarrow O_{j} \cap O_{k}=\widetilde{O}_{j k} / G_{j k}$, subject to the conditions (2.1) and (2.2). Set

$$
\mathcal{S}(O):=\left\{\chi \in \chi\left(\prod_{j} G_{j}\right): \chi_{j}\left(i_{j} g\right)=\chi_{k}\left(i_{k} g\right) \text { for all } g \in G_{j k}\right\} .
$$

It is easily verified that $\mathcal{S}(O)$ is a subgroup of the group of all characters on $\prod_{j} G_{j}$.

For an open set $U \subset O$, the restriction operator $r_{U}^{O}: \mathcal{S}(O) \rightarrow \mathcal{S}(U)$ is defined as follows. There is a finite collection of indices $J$, such that the family $\left\{O_{j}\right\}_{j \notin J}$ still covers $U$. Set

$$
r_{U}^{O} \prod_{j} \chi_{j}\left(g_{j}\right):=\prod_{j \notin J} \chi_{j}\left(g_{j}\right)
$$

for $\chi \in \mathcal{S}(O)$, thus obtaining a homomorphism of groups $\mathcal{S}(O) \rightarrow \mathcal{S}(U)$, as desired.

The presheaf $\mathcal{S}$ on $B$ constructed in this way is actually a sheaf, called the sheaf of characters. If $B$ is compact, then the group of characters $\mathcal{S}(B)$ bears full informations on $\mathcal{S}$. Indeed, for any open set $O \subset B$ the group $\mathcal{S}(O)$ has been specified as a subgroup of $\mathcal{S}(B)$.

\section{Symplectic reduction}

It is known that any compact orbifold $B$ may be obtained globally as an orbit space $B=M_{0} / G$ of a smooth manifold $M_{0}$ by a locally free action of a compact Lie group $G$ (see, for instance, [3]). If all the local groups $G_{i}$ are subgroups of $\mathrm{SO}(n)$, then $G=\mathrm{SO}(n)$ and $M_{0}$ is the space of oriented orthonormal bases in $T B$ (it is a smooth manifold). For the case when $B$ is a smooth manifold, $M_{0}$ is a principal $\mathrm{SO}(n)$-bundle over $B=M_{0} / G$. The action of $G$ is free in this case, so the base is also smooth. 
For the case of symplectic orbifolds, $M_{0}$ is a manifold of unitary bases (as described in Theorem 2.1) and $G=\mathrm{U}(n)$. Let us describe this construction in more detail. We use the notation $G$ for $\mathrm{U}(n), \mathcal{G}$ for its Lie algebra $u(n), \mathcal{G}^{*}$ for a dual space to $\mathcal{G}$ (which, of course, may be thought of as the $\mathcal{G}$ itself for the case of $\mathrm{U}(n))$. We use the notation $g^{-1}$ instead of $g^{*}$, the star is reserved for pull-backs.

Consider a smooth manifold $G \times \widetilde{O}_{i}$. To obtain the space of unitary bases over $\widetilde{O}_{i}$, we need to identify the points $(g, \tilde{x})$ under the action of $G_{i}$, namely

$$
(g, \tilde{x}) \sim\left(g \gamma^{-1}, \gamma \tilde{x}\right)
$$

for $\gamma \in G_{i}$. That is, consider the space

$$
U_{i}:=G \times{ }_{G_{i}} \widetilde{O}_{i}:=\left(G \times \widetilde{O}_{i}\right) / G_{i},
$$

the orbit space in (5.2) being taken with respect to the action (5.1). $U_{i}$ is a smooth manifold because this action is free (it acts freely on the group $G$ by $\left.g \longmapsto g \gamma^{-1}\right)$. The standard symplectic form $\omega_{B}$ on $G \times \widetilde{O}_{i}$ factors to the 2 -form on $U_{i}$ since $G_{i}$ preserves $\omega_{B}$. The group $G$ acts on $U_{i}$ from the left

$$
\gamma(g, \tilde{x})=(\gamma g, \tilde{x})
$$

commuting with the action of $G_{i}$. We thus have an action of $G$ on $U_{i}$. Moreover, we have a closed two-form $\omega_{i}$ on $U_{i}$, whose kernel coincides with the tangent subspace along the orbit. These objects glue correctly together in a manifold $M_{0}$ with the action (5.3) of $G$ and a closed two-form $\omega_{M_{0}}$ which is $G$-invariant and whose kernel coincides with the tangent space to the orbit of action (5.3).

To recover the orbifold chart $\left\{O_{i}, \widetilde{O}_{i}, G_{i}, p\right\}$ as an orbit space of the action (5.3), we fix $g=1$ in (5.1) obtaining a slice $S=(1, \tilde{x})$ of the action (5.3). Clearly, $G \cdot S=G \times \widetilde{O}_{i}$.

The stabilizer subgroup of the point $(1,0) \in U_{i}$ is precisely $G_{i}$ since for $\gamma \in G_{i}$

$$
\begin{aligned}
\gamma(1,0) & =(\gamma, 0) \\
& =(1, \gamma \cdot 0) \\
& =(1,0) .
\end{aligned}
$$

This subgroup acts on the slice $S$ since

$$
\gamma(1, \tilde{x})=(\gamma, \tilde{x}) \sim(1, \gamma \tilde{x}) .
$$

Hence the slice gives our original orbifold chart $\widetilde{O}_{i}$ with all the original structures.

We can go one step further. 
Lemma 5.1 There exists a connection form $\theta$ for the $G$-action on $M_{0}$.

Proof. Recall that by definition $\theta$ is a one-form on $M_{0}$ with values in the Lie algebra $\mathcal{G}$ of $G$ with the following properties:

$1^{o}$

$$
i\left(X_{M_{0}}\right) \theta=X
$$

the so-called reproducing property;

$2^{o}$

$$
g_{M_{0}}^{*} \theta=g \theta g^{-1}=\operatorname{Ad}_{g} \theta
$$

the equivariance property.

Here $X \in \mathcal{G}$ is an element of the Lie algebra, $X_{M_{0}}$ the corresponding vector field on $M_{0}$ (generated by the $G$-action), $g_{M_{0}}$ means the action of the group element $g \in G$ on $M_{0}$, and $g_{M_{0}}^{*}$ denotes the pull-back.

We take a right-invariant Maurer-Cartan form. For the unitary group it is given explicitly by

$$
\theta=d g g^{-1}
$$

It may be considered on the direct product $G \times \widetilde{O}_{i}$. Since the form is rightinvariant, it factors through the action (5.1) to the form on $U_{i}$. For the left action we have

$$
\gamma^{*} \theta=d(\gamma g)(\gamma g)^{-1}=\gamma\left(d g g^{-1}\right) \gamma^{-1}
$$

so that the equivariance property is also fulfilled. Hence it is correctly defined on the whole $M_{0}$.

Consider now a larger manifold

$$
M=\mathcal{G}^{*} \times M_{0}
$$

with a closed two-form

$$
\omega_{M}=-d\langle\xi, \theta\rangle+\omega_{M_{0}}
$$

Here $\mathcal{G}^{*}$ is a dual space to the Lie algebra $\mathcal{G}$ with a coadjoint action. The form $\omega_{M}$ is thus a closed $G$-invariant two-form which is non-degenerate for $\xi \in \mathcal{G}^{*}$ sufficiently small. So, after restriction to a neighborhood $O \subset \mathcal{G}^{*}$ of $\xi=0$, we obtain a symplectic manifold $M$ with an action of $G$ preserving the symplectic form ${ }^{1}$.

Lemma 5.2 The action of $G$ on $M$ is Hamiltonian with the momentum map

$$
\mu(X)=\langle\xi, X\rangle .
$$

\footnotetext{
${ }^{1}$ For a unitary group $\mathcal{G}$ and $\mathcal{G}^{*}$ with adjoint and coadjoint actions may be identified by the bilinear form $\langle\xi, X\rangle=\operatorname{tr} \xi X$
} 
Proof. For a fixed $X \in \mathcal{G}$ we have

$$
d \mu(X)=\langle d \xi, X\rangle .
$$

On the other hand, the form $\omega_{M_{0}}$ is horizontal, that is $i\left(X_{M}\right) \omega_{M_{0}}=0$, whence by $(5.8)$

$$
\begin{aligned}
i\left(X_{M}\right) \omega_{M} & =-i\left(X_{M}\right) d\langle\xi, \theta\rangle \\
& =-\mathcal{L}_{X_{M}}\langle\xi, \theta\rangle+d\left\langle\xi, i\left(X_{M}\right) \theta\right\rangle \\
& =d\langle\xi, X\rangle
\end{aligned}
$$

proving the lemma. We have used here the Cartan formula for the Lie derivative and the invariance of $\langle\xi, \theta\rangle$, so that the Lie derivative vanishes.

Summarizing, we arrive at the following theorem.

Theorem 5.3 Any compact symplectic orbifold may be obtained by a symplectic reduction of a smooth symplectic manifold by the action of the group $G \subset \mathrm{U}(n)$ by symplectomorphisms.

Moreover, our consideration shows explicitly that locally $M$ is a finite covering

$$
\mathcal{G}^{*} \times U_{i}=\mathcal{G}^{*} \times G \times \widetilde{O}_{i} / G_{i} .
$$

Observe also that $\mathcal{G}^{*} \times G=T^{*} G$ since the tangent and cotangent bundles to the Lie group manifold are always trivial.

\section{Deformation quantization}

We use the standard scheme of deformation quantization described in [4]. For symplectic orbifolds it was generalized in [14]. The scheme was discussed many times in literature, so we need not repeat it here in detail. Yet, we give a brief non-formal survey paying attention to ideas rather than to proofs.

The starting data are:

$1^{o}$ a symplectic orbifold $B$ with a symplectic connection $\partial_{B}$;

$2^{o}$ an orbifold vector bundle $E$ with a connection $\partial_{E}$.

We emphasize that the connections $\partial_{B}$ and $\partial_{E}$ are global objects (to be discussed later). They give rise to a global procedure of deformation quantization, although the construction requires local charts given by Theorems 2.1 and 3.2 .

Consider the Weyl algebra $W=W\left(\mathbb{R}^{2 n}, \omega\right)$ on a symplectic space $\mathbb{R}^{2 n}$ with a constant symplectic form $\omega=(1 / 2) \omega_{i j} d y^{i} \wedge d y^{j}$. Let $\omega^{-1}=\left(\omega^{i j}\right)$ be the 
corresponding Poisson tensor. The elements of $W$ are polynomials $a(y, h)$, with $y \in \mathbb{R}^{2 n}$ and $h \in \mathbb{R}_{+}$, the product is defined by

$$
\begin{aligned}
a(y, h) \circ b(y, h) & =\frac{1}{(\pi h)^{2 n}} \int_{\mathbb{R}^{4 n}} \exp \left(\frac{2 \imath}{h} \omega(t, \tau)\right) a(y+t, h) b(y+\tau, h) d t d \tau \\
& =\left.\exp \left(\frac{h}{2 \imath} \omega^{-1}\left(\frac{\partial}{\partial t}, \frac{\partial}{\partial \tau}\right)\right) a(y+t, h) b(y+\tau, h)\right|_{t=\tau=0} .
\end{aligned}
$$

Here the integral is understood as an oscillatory one: we insert a convergence factor $\exp \left(-\varepsilon|t|^{2}-\varepsilon|\tau|^{2}\right)$ under the integral sign and then take a limit for $\varepsilon \rightarrow 0+$. The last expression in (6.1) is the explicit value of such an integral. It should be understood as follows: we expand the exponential into a Taylor series and apply it termwise to the product $a(y+t, h) b(y+\tau, h)$ which is a polynomial in $t, \tau$. For this reason the series terminates, so the last expression is meaningful. In particular, for $a=y^{i}$ and $b=y^{j}$ we obtain

$$
y^{i} \circ y^{j}=y^{i} y^{j}+\frac{h}{2 \imath} \omega^{i j} .
$$

Thus,

$$
\left[y^{i}, y^{j}\right]=-\imath h \omega^{i j}
$$

The two expressions in (6.1) will be referred to as the Kirillov (integral) form and the Weyl (formal) form of the o-product. Observe that the Kirillov form suggests that $h$ is a fixed positive number while in the Weyl form $h$ may be a formal variable. Moreover, the Weyl form may be easily extended to formal sums of polynomials. We introduce the total degree of a monomial $h^{k} y^{\alpha}$ as $2 k+|\alpha|$ in accordance with (6.2) and (6.3), and consider formal sums

$$
a(y, h)=\sum_{k,|\alpha|=0}^{\infty} a_{k, \alpha} h^{k} y^{\alpha}
$$

having a finite number of terms with

$$
\begin{aligned}
k & \geq 0, \\
2 k+|\alpha| & \leq N,
\end{aligned}
$$

for any $N$.

We preserve the notation $W$ for the extended algebra and call it (formal) Weyl algebra. We can extend it even more, when dropping the requirement $k \geq 0$ in (6.5). The product (6.1) in the Weyl form still makes sense for this extension, which will be denoted by $W^{+}$. An example for $a \in W^{+}$not belonging to $W$ is

$$
a=\exp \left(\frac{\imath}{h} a_{i j k} y^{i} y^{j} y^{k}\right)
$$


which makes sense as formal series with the ordering by total degree.

The integral formula (6.1) may be regarded as the composition formula for (pseudo-) differential operators in the Weyl calculus. For this we need to split $\mathbb{R}^{2 n}$ into the sum $\mathbb{R}_{q}^{n} \oplus \mathbb{R}_{p}^{n}$ of two Lagrangian subspaces, so that the form $\omega$ becomes $d q \wedge d p$, and introduce a differential operator

$$
\mathrm{Op}(a(y, h)) u(q)=\frac{1}{(2 \pi h)^{n}} \int_{\mathbb{R}^{2 n}} \exp \left(\frac{\imath}{h}\left\langle q-q^{\prime}, p\right\rangle\right) a\left(\frac{q+q^{\prime}}{2}, p, h\right) u\left(q^{\prime}\right) d q^{\prime} d p .
$$

It is well known that (6.1) gives the composition rule for operators (6.6). Moreover, with fixed $h>0$ the correspondence (6.6) may be extended to functions $a(y)$ which are not polynomials, for example, to the function

$$
a(y)=\exp \left(-\frac{1}{h} \beta(y, y)\right)
$$

with a positive definite quadratic form $\beta$. For such an extension we need to use the Kirillov form of the product (6.1).

Let us observe that the Weyl correspondence (6.6) depends on the splitting $\mathbb{R}^{2 n}=\mathbb{R}_{q}^{n} \oplus \mathbb{R}_{p}^{n}$ while the composition formula does not. In particular, (6.1) is invariant with respect to linear symplectic changes of variables $y=(q, p)$.

Passing on to symplectic manifolds, we consider the Weyl bundle $W$ over $M$ whose fibers at $x \in M$ are (formal) Weyl algebras on the tangent space $T_{x} M$ (note that the symplectic form $\omega$ on $M$ defines a constant symplectic form $\omega=(1 / 2) \omega_{i j}(x) d y^{i} \wedge d y^{j}$ on each $\left.T_{x} M\right)$. The sections of $W$ are "functions" $a=a(x, y, h)$ of $x \in M$ and $y \in T_{x} M$, which at a fixed $x \in M$ are given by the formal series (6.4) (the extension $W^{+}$is also possible), and the product is given fiberwise by the Weyl form of (6.1). Clearly, the restriction $\left.W\right|_{O}$ of the Weyl bundle to an open set $O \subset M$ is defined, and the Weyl bundle itself is defined by its restrictions to any $O \subset M$. Maybe, the sheaf language is more suitable, especially when we pass on to orbifolds.

When passing on to the symplectic orbifold $B$, we define $W$ by its restrictions to orbifold charts given by Theorem 2.1. In such a chart $\{O, \widetilde{O}, G, p\}$ we define a section of $W$ by

$$
a=a(\tilde{x}, y, h)=\sum_{k,|\alpha|=0}^{\infty} h^{k} a_{k, \alpha}(\tilde{x}) y^{\alpha}
$$

with an additional invariance property

$$
a(g \tilde{x}, g y, h)=a(\tilde{x}, y, h)
$$

Here $\widetilde{x} \in \widetilde{O}$ and $y \in T_{\widetilde{x}} \widetilde{O}$. Since $g$ is a constant matrix in our chart, everything, including the formula (6.1) for the o-product, is correctly defined. 
As for the operator interpretation similar to the Weyl correspondence (6.6), it fails in general. However, it is still instructive to think of the Weyl bundle as the endomorphism bundle $\operatorname{End}(Q)$ for some imaginary, that is non-existing, "quantum" bundle $Q$.

The next step in the quantization program is to supply this "imaginary bundle" with an "imaginary connection" which is nevertheless meaningful as the connection on $W$. We define it on sections (6.7) by

$$
\partial a=d_{\tilde{x}} a+\left[\frac{\imath}{2 h} \Gamma_{i j k}^{B} y^{i} y^{j} d \tilde{x}^{k}, a\right]
$$

where $d_{\tilde{x}}$ is the de Rham differential of (6.7) with respect to $\tilde{x}$, and $\Gamma_{i j k}^{B}$ are connection coefficients of the symplectic connection $\partial^{B}$ on the orbifold $B$. We thus need to consider $W$-valued one-forms with the group action

$$
\begin{aligned}
\tilde{x} & \mapsto g \tilde{x} \\
y & \mapsto g y, \\
d \tilde{x} & \mapsto g d \tilde{x}
\end{aligned}
$$

Then the words " $\partial^{B}$ is a globally defined orbifold symplectic connection" may be interpreted in terms of (6.9) as follows:

$1^{\circ}$ The differential operator $\partial$ on sections of $W$ over $\widetilde{O}$ is a derivation of the algebra, i.e.,

$$
\partial(a \circ b)=\partial a \circ b+a \circ \partial b .
$$

$2^{\circ}$ It is invariant with respect to the group action (6.10).

$3^{\circ}$ The expression (6.9) does not depend on the orbifold chart.

The existence of such a connection, well known in the smooth case, was proved by Pflaum [14] for the orbifold case.

The form

$$
\Gamma=\frac{\imath}{2 h} \Gamma_{i j k}^{B} y^{i} y^{j} d \tilde{x}^{k}
$$

may be thought of as a local connection form for our imaginary bundle $Q$. Its definition is ambiguous: we may add any central summand $\gamma_{i} d \tilde{x}^{i}$ (not depending on $y$ ).

However, its adjoint action on section of $W$ given by (6.9) is meaningful and does not depend on the central summand. It turns out to be useful to consider the forms $\Gamma$ (6.11) normalized by $\left.\Gamma\right|_{y=0}=0$. This normalization does not depend on the chart. Moreover, being normalized, the form $\Gamma$ gives rise to a curvature form

$$
R=d \Gamma+\Gamma^{2}=\frac{\imath}{4 h} R_{i j k l}^{B} y^{i} y^{j} d \tilde{x}^{k} \wedge d \tilde{x}^{l},
$$

where $R_{i j k l}^{B}$ is the curvature tensor for the symplectic connection, so that

$$
\partial^{2} a:=\partial(\partial a)=[R, a]
$$


Again we have a situation where $R$ is the curvature of the "imaginary bundle" while its action (6.13) on $W$ is correctly defined. Thus, we come to the notion of Weyl normalization (symmetrical ordering) for connection and curvature forms. It is given by the conditions

$$
\begin{aligned}
\left.\Gamma\right|_{y=0} & =0, \\
R & =d \Gamma+\Gamma^{2} .
\end{aligned}
$$

The next step is to introduce non-trivial coefficients. Take a vector bundle $E$ with a connection $\partial^{E}$ and introduce the coefficient bundle $K=\operatorname{End}(E)$ which inherits the connection $\partial^{E}$ by adjoint representation. We consider the Weyl bundle with coefficients in $K$, that is $W \otimes K$. Its sections in linearized charts are given by matrix-valued functions (6.7) satisfying the equivariance condition

$$
a(g \tilde{x}, g y, h)=T(g) a(\tilde{x}, y, h) T(g)^{-1} .
$$

Again it is instructive to think of it as an endomorphism bundle $\operatorname{End}(Q \otimes E)$. This time the "quantum bundle" $Q \otimes E$ is partly "imaginary" ( $Q$ factor) and partly actual ( $E$ factor). The connection (6.9) on $W$ should be replaced by $\partial^{B} \otimes 1+1 \otimes \partial^{E}$ on $W \otimes K$, so that the local connection form $\Gamma$ is now

$$
\Gamma=\frac{\imath}{2 h} \Gamma_{i j k}^{B} y^{i} y^{j} d \tilde{x}^{k}+\Gamma_{k}^{E} d \tilde{x}^{k} .
$$

Here $\Gamma_{k}^{E} d \tilde{x}^{k}$ is a connection form for the bundle $E$ (an actual part which is correctly defined) and the "imaginary" part is the same as before, fixed by the Weyl normalization. The curvature becomes

$$
R=\frac{\imath}{4 h} R_{i j k l}^{B} y^{i} y^{j} d \tilde{x}^{k} \wedge d \tilde{x}^{l}+\frac{1}{2} R_{k l}^{E} d \tilde{x}^{k} \wedge d \tilde{x}^{l} .
$$

The result of the deformation quantization construction is a new connection $\mathcal{D}$ on $W \otimes K$ (or better on " $Q \otimes E$ ") with the property $\mathcal{D}^{2} a=0$. We call it Abelian, it means that its Weyl curvature is a central form. The connection $\mathcal{D}$ has the form

$$
\mathcal{D}=\partial+\left[\frac{\imath}{h} \omega_{i j} y^{i} d \tilde{x}^{j}+r, \cdot\right]
$$

with $\operatorname{deg} r \geq 1$. Under some normalizations the global one-form $r$ is uniquely determined by the requirement that its Weyl curvature is equal to

$$
\Omega=-\frac{\imath}{h} \omega_{B} .
$$

It is automatically $G$-invariant with respect to the action (6.15).

Now, let us define the subalgebra $W_{\mathcal{D}}$ of flat sections of $W \otimes K$, that is $a \in W_{\mathcal{D}}$ means that $\mathcal{D} a \equiv 0$. We call it the algebra of quantum observables. Moreover, we have a correspondence between classical and quantum 
observables. To see this, note that the classical observable is a section of $K$, $a(x) \in C^{\infty}(K)$, with the pointwise matrix multiplication. Let us extend this algebra by formal parameter $h$, so that we consider "functions" from $C^{\infty}(K)[[h]]$. In a local chart such a section is given by a "function"

$$
a(\tilde{x}, h)=\sum_{k=0}^{\infty} h^{k} a_{k}(\tilde{x}) .
$$

On the other hand, a quantum observable is given by a "function"

$$
b(\tilde{x}, y, h)=\sum_{k,|\alpha|=0}^{\infty} h^{k} b_{k, \alpha}(\tilde{x}) y^{\alpha} .
$$

Both functions satisfy the corresponding equivariance condition and $b$ also satisfies $\mathcal{D} b=0$.

The final result in our construction is the following statement.

Theorem 6.1 There exists a one-to-one correspondence between flat sections (6.21) and sections (6.20), given by

$$
\sigma: b(\tilde{x}, y, h) \mapsto b(\tilde{x}, 0, h) .
$$

The inverse map $Q$, called quantization map, gives for any classical observable $a(x) \in C^{\infty}(K)$ a flat section $b=Q a$ such that

$$
\left.(Q a)\right|_{y=0}=a \text {. }
$$

Using the maps $\sigma$ and $Q$, we may transport the $\circ$-product of flat sections to a product defined directly on functions $a(\tilde{x}, h)$ as follows

$$
a(\tilde{x}, h) * b(\tilde{x}, h)=\sigma(Q a \circ Q b) .
$$

We will use both $\circ$-product of flat sections and $*$-product of functions.

This completes the global part. More detailed study requires restriction to a linearized chart and subsequent operator representation similar to the Weyl correspondence.

\section{Orbifold trace}

To define the trace, we have to consider operator representations of the quantum algebra $W$ restricted to an orbifold chart $\{O, \widetilde{O}, G, p\}$.

So, let us consider the subalgebra $\left.W_{\mathcal{D}}\right|_{\tilde{O}}$ of flat sections $a(\tilde{x}, y, h)$ with supports in $\widetilde{O}$, that is $a \equiv 0$ if $\tilde{x}$ is outside a compact subset of $\widetilde{O}$. The 
sections also satisfy equivariance conditions (6.15) with the linear action of the finite group $G \subset \mathrm{U}(n) \otimes \mathrm{U}(N)$, where $\mathrm{U}(N)$ stands for the action of $G$ in fibers of the bundle $E$. Suppose for a moment that we have constructed a representation of the algebra $\left.W_{\mathcal{D}}\right|_{\tilde{O}}$ and the group $G$ by operators in a Hilbert space $H$ with the following properties:

$1^{o}$ a homomorphism property

$$
\begin{aligned}
\hat{a} \hat{b} & =\widehat{a \circ b}, \\
\hat{g}_{1} \hat{g}_{2} & =\widehat{g_{1} g_{2}} ;
\end{aligned}
$$

$2^{o}$ a conjugation property

$$
\hat{g} \hat{a} \widehat{a g^{-1}}=\widehat{g^{*}} a .
$$

Here hats mean the corresponding operators in $H$, and

$$
\left(g^{*} a\right)(\tilde{x}, y, h)=T(g)^{-1} a(g \tilde{x}, g y, h) T(g)
$$

is the pull-back of the section $a$ by the group action.

Then we define (locally) the orbifold trace by

$$
\operatorname{Tr}_{\text {orb }} a=\left.\operatorname{Tr} \hat{a}\right|_{\text {inv }},
$$

$\left.\hat{a}\right|_{\text {inv }}$ on the right-hand side meaning an operator restricted to the subspace of $G$-invariant elements of $H$. For a finite group $G$, the orthogonal projector to this subspace is given by the averaging

$$
P_{\mathrm{inv}} u=\frac{1}{|G|} \sum_{g \in G} \hat{g} u,
$$

and (7.4) thus becomes

$$
\operatorname{Tr}_{\text {orb }} a=\frac{1}{|G|} \sum_{g \in G} \operatorname{Tr} \hat{g} \hat{a} .
$$

Our aim is to express each summand directly in terms of deformation quantization, although the construction itself (see [8]) requires an operator representation. We recall it briefly paying special attention to the invariance properties. We will also use the notation $\operatorname{Tr}_{g} a$ for the summands of (7.5) to stress their deformation quantization nature.

At the first step we construct an isomorphism to the Weyl algebra. In a linearized chart the de Rham differential defines connections $\partial^{B}$ and $\partial^{E}$ for which the corresponding connection forms $\Gamma^{B}$ and $\Gamma^{E}$ are equal to 0 . In this 
case the deformation quantization construction of the previous section leads to the Abelian connection

$$
\mathcal{D}_{0}=d+\left[\frac{\imath}{h} \omega_{i j} y^{i} d x^{j}, \cdot\right]
$$

with the same Weyl curvature (6.19). The quantization map for this connection looks extremely simple:

$$
Q a(\tilde{x})=a(\tilde{x}+y):=\sum_{|\alpha|=0}^{\infty} \frac{1}{\alpha !} a^{(\alpha)}(\tilde{x}) y^{\alpha},
$$

and the algebra $\left.W_{\mathcal{D}_{0}}\right|_{\widetilde{O}}$ consists of the formal Taylor series of matrix-valued functions (7.6).

The following lemma describes the needed isomorphism $[4,5]$.

Lemma 7.1 There exists a section

$$
\begin{aligned}
U(\tilde{x}, y, h) & =1-\frac{\imath}{6 h} \Gamma_{i j k}^{B} y^{i} y^{j} y^{k}-\Gamma_{k}^{E} y^{k}+\ldots \\
& \in C^{\infty}\left(W^{+} \otimes K\right)
\end{aligned}
$$

such that for any flat section $\left.a \in W_{\mathcal{D}}\right|_{\widetilde{O}}$ its image under the conjugation

$$
a_{0}=I a=U \circ a \circ U^{-1}
$$

belongs to $\left.W_{\mathcal{D}_{0}}\right|_{\widetilde{O}}$.

Consider now a homotopy $f_{t}(\tilde{x})$ of a linearized symplectic chart constructed in Theorem 2.2. The change of variables $f_{t}(\tilde{x})$ and the conjugation isomorphism (7.7) applied to a flat section $\left.a \in W_{\mathcal{D}}\right|_{\widetilde{O}}$ give a family of flat sections of $\left.W_{\mathcal{D}_{0}}\right|_{\widetilde{O}}$

$$
\begin{aligned}
a_{0}(\tilde{x}+y, h, t) & =I_{t} a(\tilde{x}, y, h) \\
& =U_{t} \circ a\left(f_{t}(\tilde{x}), f_{t}^{\prime}(\tilde{x}) y, h\right) \circ U_{t}^{-1} \\
& \in W_{\mathcal{D}_{0}} \mid \tilde{O} .
\end{aligned}
$$

Differentiating in $t$, we come to the following Heisenberg equation (for more detail see [5, Section 5.4])

$$
\dot{a}_{0}=\left[H, a_{0}\right]
$$

where $H$ is a section of $W^{+} \otimes K$ (depending on $t$ ).

Lemma 7.2 There exists a flat section $H_{0} \in W_{\mathcal{D}_{0}}$ such that (7.8) may be rewritten in the form

$$
\dot{a}_{0}=\left[\frac{\imath}{h} H_{0}, a_{0}\right] .
$$


Proof. Applying $\mathcal{D}_{0}$ to both sides of (7.8) and using the fact that $a_{0}, \dot{a}_{0}$ are flat with respect to $\mathcal{D}_{0}$, we get

$$
\left[\mathcal{D}_{0} H, a_{0}\right]=0 \text {. }
$$

Being valid for any flat section $a_{0}$, this equality implies that $\mathcal{D}_{0} H$ is a central one-form, that is independent of $y$. This yields

$$
\mathcal{D}_{0} H=\varphi=\varphi_{i}(\tilde{x}, h, t) d \tilde{x}^{i} .
$$

Applying $\mathcal{D}_{0}$ to both sides, we get 0 on the left since $\mathcal{D}_{0}\left(\mathcal{D}_{0} H\right) \equiv 0$. On the other hand, $\mathcal{D}_{0} \varphi=d \varphi$ since $\varphi$ is a central form. Hence, $\varphi=d \psi$ in the local chart. It means that the section $\widetilde{H}=H-\psi$ belongs to $W_{\mathcal{D}_{0}}$. Clearly, the section $H$ in (7.8) may be replaced by $\widetilde{H}$, for they differ by a central function $\psi(\tilde{x}, h, t)$.

Comparing degrees on both sides of (7.8), one can see that $\widetilde{H}$ must have degree -2 , thus it can be rewritten in the form $\imath / h H_{0}$ with $H_{0} \in W_{\mathcal{D}_{0}}$.

Now, putting $y=0$, we conclude that $W_{\mathcal{D}_{0}}$ is isomorphic to a standard Weyl algebra on $\mathbb{R}^{2 n}$. The Weyl product $\circ$ with respect to variables $y$ goes to the Weyl product with respect to $\tilde{x}$ which will be denoted by $*$. Using a complex structure and writing $\tilde{x}=(z, \bar{z})$ with $z \in \mathbb{C}^{n}$, we rewrite the $*$-product in the complex form

$$
\begin{aligned}
& a(z, \bar{z}, h) * b(z, \bar{z}, h) \\
& =\frac{1}{(\pi h)^{2 n}} \int_{\mathbb{C}^{2 n}} \exp \left(\frac{1}{h}\left(u^{*} v-v^{*} u\right)\right) a(z+u, \bar{z}+\bar{u}, h) b(z+v, \bar{z}+\bar{v}, h) d u d \bar{u} d v d \bar{v} \\
& =\sum_{\alpha, \beta}(-1)^{|\beta|} \frac{h^{|\alpha|+|\beta|}}{\alpha ! \beta !} \frac{\partial^{\alpha+\beta} a}{\partial z^{\alpha} \partial \bar{z}^{\beta}} \frac{\partial^{\alpha+\beta} b}{\partial \bar{z}^{\alpha} \partial z^{\beta}} .
\end{aligned}
$$

Again we have two expressions for the $*$-product. The integral (Kirillov) form suggests that $h$ is a positive number, $a$ and $b$ are polynomials in $z, \bar{z}$. The notation $d u d \bar{u}$ means the usual Lebesgue measure in $\mathbb{C}^{n}, u, v, z$ are often treated as column vectors, then $u^{*}, v^{*}, z^{*}$ mean the rows of complex conjugate elements, so that $v^{*} u=\bar{v}_{1} u_{1}+\ldots+\bar{v}_{n} u_{n}$ is a Hermitian scalar product. The last expression in (7.10) gives the explicit value of the integral. This last expression may be extended to the formal Weyl algebra.

At the second step we construct the representation of the Weyl algebra $W_{\widetilde{O}}$ and the group $G \in \mathrm{U}(n)$ in the Fock space. Here by Weyl algebra we mean the algebra of smooth functions on $\widetilde{O}$ with the $*$-product in the integral form for a fixed positive $h$. Thus, there is the Weyl correspondence between such 
functions (Weyl symbols) and pseudodifferential operators in $L^{2}\left(\mathbb{R}^{n}\right)$ defined by (6.6). Unfortunately, the group $\mathrm{U}(n)$ can not be represented in $L^{2}\left(\mathbb{R}^{n}\right)$ in a single-valued manner, this is why we need a Fock space representation. Consider an operator on $L^{2}\left(\mathbb{R}^{n}\right)$ with the Weyl symbol

$$
p(z, \bar{z})=2^{n} \exp \left(-\frac{1}{h}|z|^{2}\right)
$$

with a fixed positive $h$. The integral form of the $*$-product shows that $p * p=p$. It means that the operator $P=\mathrm{Op}(p)$ is a projector in $L^{2}\left(\mathbb{R}^{n}\right)$. Furthermore, we have

$$
\operatorname{Tr} P=\frac{1}{(2 \pi h)^{n}} \int_{\mathbb{C}^{n}} p(z, \bar{z}) d z d \bar{z}=1,
$$

which means that $P$ is a one-dimensional projector. Now, using again the integral form, we see that

$$
\begin{aligned}
& z^{i} * p=0 \\
& \bar{z}^{i} * p \neq 0
\end{aligned}
$$

For this reason $z^{i}$ are called annihilation operators while $\bar{z}^{i}$ creation operators. As a linear space the Fock space is generated by the symbols

$$
u=a(z, \bar{z}) * p(z, \bar{z})
$$

where $a(z, \bar{z})$ are polynomials in $z, \bar{z}$.

Define the scalar product

$$
(u, v)=\frac{1}{(2 \pi h)^{n}} \int_{\mathbb{C}^{n}} p * \bar{b} * a * p d z d \bar{z}
$$

for $u=a * p$ and $v=b * p$. Writing $a(z, \bar{z})$ in (7.12) in the normal form $a_{\alpha, \beta} \bar{z}^{\alpha} * z^{\beta}$ (first creation, then annihilation operators), we see that non-zero vectors have the form

$$
e_{\alpha}=\frac{\bar{z}^{\alpha} * p}{\sqrt{\alpha !(2 h)^{|\alpha|}}}
$$

and they form an orthonormal basis with respect to the scalar product (7.13). The vector $e_{0}=p$ is called a vacuum. Thus, the Fock space $\mathbb{F}$ is the left ideal of the symbol algebra $W$ generated by the vacuum vector. To obtain a Hilbert space, we need a completion with respect to the scalar product.

On $\mathbb{F}$ we have a natural action of the symbol algebra $W$ and the group $\mathrm{U}(n)$. For a symbol $b(z, \bar{z})$ we define an operator $\hat{b}$ which acts on vectors (7.12) as

$$
\hat{b} u=b(z, \bar{z}) * a(z, \bar{z}) * p .
$$

The group $\mathrm{U}(n)$ acts by pulling back the symbols (7.12) which represent vectors in $\mathbb{F}$. On the vacuum vector, the action is trivial, for

$$
\hat{g} e_{0}=g^{*}(p(z, \bar{z}))=p(g z, \overline{g z})=p(z, \bar{z}) .
$$


Then

$$
\hat{g} u=g^{*}(a(z, \bar{z}) * p(z, \bar{z}))=a(g z, \overline{g z}) * p(z, \bar{z}) .
$$

It is easy to see that the properties (7.1), (7.2) are fulfilled literary if $a, b \in W$ are polynomials. But the representation (7.15) still makes sense for more general symbols $b(z, \bar{z})$, e.g., for compactly supported functions. Of course, in this case we need the integral expression for the $*$-product.

The final step in the definition of the $\operatorname{Tr}_{g} a$ is now obvious. For a symbol $a(z, \bar{z}) \in C^{\infty}(\widetilde{O}, K)$, consider the corresponding operator $\hat{a}$ in the Fock space and set

$$
\begin{aligned}
\operatorname{Tr}_{g} a & =\operatorname{Tr} \hat{g} \hat{a} \\
& =\sum_{\alpha}\left(\hat{g} \hat{a} e_{\alpha}, e_{\alpha}\right) .
\end{aligned}
$$

The sum may be calculated explicitly (see [8])

$$
\operatorname{Tr} \hat{g} \hat{a}=\frac{1}{(\pi h)^{n}} \frac{1}{\operatorname{det}(1+g)} \int_{\mathbb{C}^{n}} \exp \left(-\frac{1}{h} z^{*} \frac{1-g}{1+g} z\right) \operatorname{tr} T(g) a(z, \bar{z}) d z d \bar{z},
$$

provided $\operatorname{det}(1+g) \neq 0$. Here $T(g)$ means the action of $G$ on the bundle $E, \operatorname{tr}$ means the coefficient trace. This is the Kirillov (integral) form of the trace formula. To extend it to the formal Weyl algebra, we need to calculate a stationary phase expansion of the integral. To this end decompose $\mathbb{C}^{n}$ into the direct sum of the fixed point subspace

$$
\widetilde{F}(g)=\operatorname{ker}(1-g)
$$

and its orthogonal complement $N(g)$. Writing $z=\left(z_{1}, z_{2}\right), n_{1}=\operatorname{dim} \widetilde{F}(g)$, $n_{2}=\operatorname{dim} N(g)$, and $g=1$ on $\widetilde{F}(g)$ and $g=g_{2}$ on $N(g)$, we get

$$
\begin{aligned}
& \operatorname{Tr} \hat{g} \hat{a}=\frac{1}{(2 \pi h)^{n_{1}}} \int_{\widetilde{F}(g)} d z_{1} d \bar{z}_{1} \\
& \quad \frac{1}{(\pi h)^{n_{2}}} \frac{1}{\operatorname{det}\left(1+g_{2}\right)} \int_{N(g)} \exp \left(-\frac{1}{h} z_{2}^{*} \frac{1-g_{2}}{1+g_{2}} z_{2}\right) \operatorname{tr} T(g) a\left(z_{1}, \bar{z}_{1}, z_{2}, \bar{z}_{2}\right) d z_{2} d \bar{z}_{2} .
\end{aligned}
$$

To calculate the inner integral, we expand $a$ in a formal Taylor series at $z_{2}=0$, namely

$$
a\left(z_{1}, \bar{z}_{1}, z_{2}, \bar{z}_{2}\right)=\sum_{\alpha, \beta} \frac{1}{\alpha ! \beta !} \frac{\partial^{\alpha+\beta}}{\partial z_{2}^{\alpha} \partial \bar{z}_{2}^{\beta}} a\left(z_{1}, \bar{z}_{1}, 0,0\right) z_{2}^{\alpha} \bar{z}_{2}^{\beta},
$$

and integrate this expansion termwise, thus obtaining

$$
\left.\frac{1}{\operatorname{det}\left(1-g_{2}\right)} \exp \left(h \frac{\partial}{\partial z_{2}} \frac{1+g_{2}}{1-g_{2}} \frac{\partial}{\partial z_{2}^{*}}\right) \operatorname{tr} T(g) a\left(z_{1}, \bar{z}_{1}, z_{2}, \bar{z}_{2}\right)\right|_{z_{2}=\bar{z}_{2}=0} .
$$


Here $\partial / \partial z_{2}$ means the row $\left(\partial / \partial z_{2}^{1}, \ldots, \partial / \partial z_{2}^{n_{2}}\right)$ and $\partial / \partial z_{2}^{*}$ the column of complex conjugate elements. Observe also that on $N(g)$ the matrix $1-g_{2}$ is non-degenerate.

Consider further the symplectic form

$$
\omega_{\widetilde{F}(g)}=\frac{1}{2 \imath}\left(d \bar{z}_{1}^{1} \wedge d z_{1}^{1}+\ldots+d \bar{z}_{1}^{n_{1}} \wedge d z_{1}^{n_{1}}\right) .
$$

The measure $d z_{1} d \bar{z}_{1}$ including the factor $(2 \pi h)^{n_{1}}$ in the outer integral is defined by the top degree of the non-homogeneous form

$$
\exp \left(\frac{\omega_{\widetilde{F}(g)}}{2 \pi h}\right)
$$

In the final expression we denote $\left(z_{1}, \bar{z}_{1}\right)$ by $\tilde{x},\left(z_{2}, \bar{z}_{2}\right)$ by $(z, \bar{z})$ and $g_{2}$ by $g_{N}$. The trace formula reduces then to the so-called Weyl form

$$
\begin{aligned}
& \operatorname{Tr}_{g} a \\
& =\left.\int_{\widetilde{F}(g)} \exp \left(\frac{\omega_{\widetilde{F}(g)}}{2 \pi h}\right) \frac{1}{\operatorname{det}\left(1-g_{N}\right)} \exp \left(h \frac{\partial}{\partial z} \frac{1+g_{N}}{1-g_{N}} \frac{\partial}{\partial z^{*}}\right) \operatorname{tr} T(g) a(\tilde{x}, z, \bar{z})\right|_{z=\bar{z}=0} .
\end{aligned}
$$

This expression is meaningful for the formal Weyl algebra, and we take it as a definition of $\operatorname{Tr}_{g} a$.

Our next goal is to prove that this local formula is independent of a linearized chart. The proof is based on the local properties of $\operatorname{Tr}_{g} a$, considered in [8]. Nevertheless, we are going to give a proof for the main property since the reasoning here is typical when one deals with deformation quantization via operator representations.

Lemma 7.3 The following relation holds

$$
\operatorname{Tr}_{g} a * b=\operatorname{Tr}_{g} b *\left(g^{*} a\right) .
$$

Proof. The operator counterpart of (7.20) is

$$
\operatorname{Tr} \hat{g} \hat{a} \hat{b}=\operatorname{Tr} \hat{g} \hat{b} \widehat{g^{*} a} .
$$

This equation is considered in the Fock space for a positive $h$ and for operators defined by Weyl symbols $a(z, \bar{z})$ and $b(z, \bar{z})$, one of them (say $a$ ) being a polynomial and another having compact support. Certainly, it is true since

$$
\operatorname{Tr} \hat{g} \hat{a} \hat{b}=\operatorname{Tr} \hat{g} \hat{a} \widehat{g^{-1}} \hat{g} \hat{b}=\operatorname{Tr} \hat{g} \hat{b} \widehat{b} \widehat{g^{*}} .
$$

We have used (7.2) and changed cyclically the order of factors under the trace sign. 
Further, the operator identity (7.21) may be rewritten as a symbol identity (7.20) with the integral form of $*$-product. Taking stationary phase approximations, we obtain an asymptotic identity for symbols. Under our assumptions on $a$ and $b$ the integral form of the $*$-product reduces then to the Weyl form where $h$ is still treated as a positive number and the series as asymptotic ones. Being valid in asymptotic sense, the identity (7.20) holds true in the sense of formal series but still for our special symbols $a$ and $b$. Finally, we can extend it by linearity to any formal Weyl symbols

$$
\begin{aligned}
a & =\sum_{k,|\alpha|=0}^{\infty} h^{k} a_{k, \alpha} \tilde{x}^{\alpha}, \\
b & =\sum_{k} h^{k} b_{k}(\tilde{x})
\end{aligned}
$$

with compactly supported $b_{k}$.

We mention also without proof (see [8]) that $\operatorname{Tr}_{g} a$ does not depend on the positive complex structure $J$ needed for the Fock space construction.

This lemma has many important consequences.

Corollary 7.4 If one of the symbols is $g$-invariant, that is $g^{*} a=a$, then

$$
\operatorname{Tr}_{g} a * b=\operatorname{Tr}_{g} b * a
$$

Corollary 7.5 The functional $\operatorname{Tr}_{g}$ a on $g$-invariant symbols a with compact support is independent of the choice of a linearized symplectic chart and a positive complex structure.

Proof. First of all, it follows from (7.19) that a linear change $z \mapsto u z$, where $u \in \mathrm{U}(n)$, together with the conjugation $g \mapsto u g u^{-1}$ does not change $\operatorname{Tr}_{g} a$. Besides, the choice of a positive complex structure is also inessential. So, we need to consider only homotopies $f_{t}(\tilde{x})$ (cf. Theorem 2.2) of a linearized symplectic chart with identical linear parts. Using Lemma 7.2, we get

$$
\operatorname{Tr}_{g} \dot{a}=\frac{\imath}{h} \operatorname{Tr}_{g}\left[H_{0}, a\right] .
$$

Since $a$ is a $g$-invariant symbol with compact support and $H_{0}$ belongs to the formal Weyl algebra, the right-hand side vanishes by Corollary 7.4, proving that $\operatorname{Tr}_{g} a$ is independent of the choice of a linearized chart.

This finishes the trace construction for quantum observables with a support in an orbifold chart. Our next goal is to combine the local expressions into a global formula. 
We begin with a global formula for the orbifold trace of a flat section $a \in W_{\mathcal{D}}$. To this end, we first construct a partition of unity in the algebra $W_{\mathcal{D}}$. We take a partition of unity $\left\{\rho_{i}(x)\right\}$ of the space $B$ subordinate to a covering $\left\{O_{i}, \widetilde{O}_{i}, G_{i}, p_{i}\right\}$ by orbifold charts. When lifting $\rho_{i}$ to $\widetilde{O}_{i}$, we get a $G_{i}$-invariant smooth function $\rho_{i}(\tilde{x})$, and we quantize it obtaining a flat section $Q \rho_{i} \in W_{\mathcal{D}}$. Since deformation quantization is a global procedure, these sections actually give us the needed partition of unity, i.e.

$$
a=\sum_{i}\left(Q \rho_{i}\right) \circ a
$$

for any flat section $a$. The summands in this decomposition are flat sections with supports in $\widetilde{O}_{i}$. Next, we apply the isomorphism $I_{i}$ defined by (7.7) obtaining a flat section $I_{i}\left(Q \rho_{i} \circ a\right)$ in $\left.W_{\mathcal{D}_{0}}\right|_{\widetilde{O}_{i}}$. And putting $y=0$, we get a function $a_{i}(\tilde{x}, h)$, that is a Weyl symbol with values in $K$ on the standard symplectic space $\mathbb{R}^{2 n}$ :

$$
\begin{aligned}
a_{i}(\tilde{x}, h) & =\left.I_{i}\left(Q \rho_{i} \circ a\right)\right|_{y=0} \\
& \in W\left(\mathbb{R}^{2 n}, K\right) .
\end{aligned}
$$

Taking local traces (7.19) and gathering all together, we come to the global trace formula

$$
\begin{aligned}
& \operatorname{Tr}_{\text {orb }} a=\sum_{i} \operatorname{Tr}_{\text {orb }} a_{i}=\sum_{i} \frac{1}{\left|G_{i}\right|} \sum_{g \in G_{i}} \\
&\left.\int_{\widetilde{F}(g)} \exp \left(\frac{\omega_{\widetilde{F}(g)}}{2 \pi h}\right) \frac{1}{\operatorname{det}\left(1-g_{N}\right)} \exp \left(h \frac{\partial}{\partial z} \frac{1+g_{N}}{1-g_{N}} \frac{\partial}{\partial z^{*}}\right) \operatorname{tr} T_{i}(g) a_{i}(\tilde{x}, z, \bar{z})\right|_{z=\bar{z}=0} .
\end{aligned}
$$

What we would like to emphasize is that the choice of isomorphisms $I_{i}$ in (7.23) is irrelevant as was proved in the preceding section, we could take instead any isomorphism $I$ to a local linearized symplectic chart. In other words, equation (7.25) gives a global quantity expressed in local terms.

We are going now to transform this formula to a more invariant form. Note that the inner sum (over $g \in G_{i}$ ) contains many equal summands. Indeed, the integrals over $\widetilde{F}(g)$ and $\widetilde{F}\left(\gamma g \gamma^{-1}\right)=\gamma \widetilde{F}(g)$ are the same because of $G_{i}$ ivariance of the integrand. Thus, decomposing $G_{i}$ into a union of conjugacy classes and choosing a representative $g_{k}$ in each conjugacy class $\left(g_{k}\right)$, we can rewrite the inner sum as

$$
\sum_{\left(g_{k}\right) \in\left(G_{i}\right)} \frac{\left|\left(g_{k}\right)\right|}{\left|G_{i}\right|} \int_{\widetilde{F}\left(g_{k}\right)} \ldots
$$


Here $\left(G_{i}\right)$ denotes the set of conjugacy classes of $G_{i}, g_{k}$ runs over the whole set of representatives, and $\left|\left(g_{k}\right)\right|$ denotes the number of elements in the conjugacy class. We have dropped the integrand, it is the same as in (7.25) with $g$ replaced by $g_{k}$. To calculate the number $\left|\left(g_{k}\right)\right|$, observe that the elements $\gamma_{1} g_{k} \gamma_{1}^{-1}$ and $\gamma_{2} g_{k} \gamma_{2}^{-1}$ coincide if and only if $\gamma_{1} \gamma_{2}^{-1}$ commutes with $g_{k}$, that is belongs to the centralizer of $g_{k}$ in $G_{i}$. (Recall that the centralizer $Z_{G_{i}}\left(g_{k}\right)$ is a subgroup in $G_{i}$ consisting of elements commuting with $g_{k}$ ). To obtain all distinct elements $\gamma g_{k} \gamma^{-1}$, the element $\gamma$ should run over all representatives of left cosets $G_{i} / Z_{G_{i}}\left(g_{k}\right)$. Thus,

$$
\left|\left(g_{k}\right)\right|=\frac{\left|G_{i}\right|}{\left|Z_{G_{i}}\left(g_{k}\right)\right|}
$$

and the sum takes the form

$$
\sum_{\left(g_{k}\right) \in\left(G_{I}\right)} \frac{1}{\left|Z_{G_{i}}\left(g_{k}\right)\right|} \int_{\widetilde{F}\left(g_{k}\right)} \ldots
$$

The summands here may be interpreted as integrals over linearized charts of some symplectic orbifolds. Indeed, $\widetilde{F}\left(g_{k}\right)=\operatorname{ker}\left(1-\left(g_{k}\right)_{N}\right)$ is a complex space where the group $Z_{G_{i}}\left(g_{k}\right)$ acts by linear unitary transformations since

$$
\gamma \widetilde{F}\left(g_{k}\right)=\widetilde{F}\left(\gamma g_{k} \gamma^{-1}\right)=\widetilde{F}\left(g_{k}\right) .
$$

Thus, introducing the notation $F\left(g_{k}\right)$ for the orbit space $\widetilde{F}\left(g_{k}\right) / Z_{G_{i}}\left(g_{k}\right)$ and $p\left(g_{k}\right)$ for the corresponding projection, we come to a quadruple

$$
\left\{F\left(g_{k}\right), \widetilde{F}\left(g_{k}\right), Z_{G_{i}}\left(g_{k}\right), p\left(g_{k}\right)\right\}
$$

which resembles an orbifold chart for some symplectic orbifold. In fact there are many connected symplectic orbifolds of different dimensions (and even for a given dimension there are many connected components) whose orbifold charts form the set (7.26) with all possible $k$ and $i$. We will denote these connected components by $F_{1}, F_{2}, \ldots, F_{m}$ and call them fixed point orbifolds. To define these orbifolds completely, we need to indicate what pairs from the set (7.26) should be glued. Each chart (7.26) is contained in a chart $\widetilde{O}_{i}$ of the original orbifold $B$. If $O_{i} \cap O_{j}=\emptyset$ then, clearly, there is no gluing conditions for the charts $\widetilde{F}\left(g_{k}\right) \in \widetilde{O}_{i}$ and $\widetilde{F}\left(g_{l}\right) \in \widetilde{O}_{j}$. Otherwise, if $O=O_{i} \cap O_{j} \neq \emptyset$, we glue $F\left(g_{k}\right) \in O_{i}$ and $F\left(g_{l}\right) \in O_{j}$ if and only if $F\left(g_{k}\right) \cap O=F\left(g_{l}\right) \cap O$. A connected component of the fixed point orbifold consist of those charts from the set (7.26) which can be connected with each other by a chain of pairwise glued charts (7.26).

The action of the centralizer $Z_{G_{i}}\left(g_{k}\right)$ on $\widetilde{F}\left(g_{k}\right)$ may be not effective, that is the components $F_{k}$ may have a multiplicity $m\left(F_{k}\right)>1$, even if the multiplicity 
of the original orbifold $B$ was equal to 1 . Then by the integration formula $(3.4)$

$$
\operatorname{Tr}_{\text {orb }} a=\sum_{k} \frac{1}{m\left(F_{k}\right)} \int_{F_{k}} \alpha_{k}
$$

where the integrand $\alpha_{k}$ is a differential form on $F_{k}$ which in an orbifold chart $\widetilde{F}(g)$ is defined by

$$
\alpha=\left.\exp \left(\frac{\omega_{\widetilde{F}(g)}}{2 \pi h}\right) \frac{1}{\operatorname{det}\left(1-g_{N}\right)} \exp \left(h \frac{\partial}{\partial z} \frac{1+g_{N}}{1-g_{N}} \frac{\partial}{\partial z^{*}}\right) \operatorname{tr} T_{i}(g) a_{i}(\tilde{x}, z, \bar{z})\right|_{z=\bar{z}=0} .
$$

Lemma 7.6 The functional

$$
\left.\operatorname{Tr}_{\text {orb }}: W_{\mathcal{D}}^{c} \rightarrow \mathbb{C}\left[h^{-1}, h\right]\right]
$$

possesses the trace property

$$
\operatorname{Tr}_{\text {orb }} a \circ b=\operatorname{Tr}_{\text {orb }} b \circ a,
$$

where $W_{\mathcal{D}}^{c} \subset W_{\mathcal{D}}$ means the ideal of flat sections with compact supports.

Proof. The trace property follows from Corollaries 7.4 and 7.5 in virtue of the chain of equalities

$$
\begin{aligned}
\operatorname{Tr}_{\text {orb }} a \circ b & =\sum_{i, j} \operatorname{Tr}_{\text {orb }}\left(Q \rho_{i}\right) \circ a \circ\left(Q \rho_{j}\right) \circ b \\
& =\left.\sum_{i, j} \sum_{g \in G_{i}} \frac{1}{\left|G_{i}\right|} \operatorname{Tr}_{g} I_{i}\left(\left(Q \rho_{i}\right) \circ a \circ\left(Q \rho_{j}\right) \circ b\right)\right|_{y=0} \\
& =\left.\left.\sum_{i, j} \sum_{g \in G_{i}} \frac{1}{\left|G_{i}\right|} \operatorname{Tr}_{g} I_{i}\left(\left(Q \rho_{i}\right) \circ a\right)\right|_{y=0} * I_{i}\left(\left(Q \rho_{j}\right) \circ b\right)\right|_{y=0} \\
& =\left.\left.\sum_{i, j} \sum_{g \in G_{i}} \frac{1}{\left|G_{i}\right|} \operatorname{Tr}_{g} I_{i}\left(\left(Q \rho_{j}\right) \circ b\right)\right|_{y=0} * I_{i}\left(\left(Q \rho_{i}\right) \circ a\right)\right|_{y=0} \\
& =\left.\sum_{i, j} \sum_{g \in G_{i}} \frac{1}{\left|G_{i}\right|} \operatorname{Tr}_{g} I_{i}\left(\left(Q \rho_{j}\right) \circ b \circ\left(Q \rho_{i}\right) \circ a\right)\right|_{y=0} \\
& =\left.\sum_{i, j} \sum_{g \in G_{j}} \frac{1}{\left|G_{j}\right|} \operatorname{Tr}_{g} I_{j}\left(\left(Q \rho_{j}\right) \circ b \circ\left(Q \rho_{i}\right) \circ a\right)\right|_{y=0} \\
& =\operatorname{Tr}_{\mathrm{orb}} b \circ a .
\end{aligned}
$$

The summation, of course, runs over those pairs $i, j$ for which the intersection $O_{i} \cap O_{j}$ is non-empty. 
In contrast to the smooth case the orbifold trace is not unique. The matter is that the action of the group $G_{i}$ on the Fock space is not uniquely defined. In fact, the action on the vacuum may be non-trivial. In general we may replace (7.16) by any one-dimensional representation, that is

$$
\hat{g} e_{0}=\chi_{i}(g) e_{0},
$$

with a one-dimensional character $\chi_{i}$. This factor would appear in all the subsequent formulas, giving another orbifold trace which may be obtained from equations $(7.27)$, (7.28) by replacing $T_{i}(g)$ by $\chi_{i}(g) T_{i}(g)$ in (7.28). Globally these factors define a section of the sheaf of characters, that is a onedimensional flat orbifold vector bundle considered in Section 4 under the name of the Picard group. Clearly, the new orbifold trace may be obtained from the old one by tensoring the original vector bundle $E$ by $E_{0} \in \operatorname{Pic}(B)$. This tensoring does not affect the structure of the algebra $W_{\mathcal{D}}$ since $E_{0}$ is flat and $\operatorname{End}\left(E_{0}\right)$ is commutative, but the trace will be different. It is plausible that this ambiguity in traces is the only possible one. Unfortunately, at the moment we can not prove this fact.

Summarizing, we arrive at the following theorem.

Theorem 7.7 There exist trace functionals (7.29) on the algebra $W_{\mathcal{D}}$ satisfying a trace property (7.30). The Picard group acts on the set of traces by tensoring.

One of the components in (7.27) coincides with the original orbifold $B$ whose multiplicity is equal to 1 according to the assumption in Section 2. This case corresponds to the conjugacy class (1) of identity element in any local group $G_{i}$, the centralizer is then the whole group $G_{i}$. For $g=1$ the integrand (7.28) takes a more simple form

$$
\alpha=\exp \left(\frac{\omega}{2 \pi h}\right) \operatorname{tr} a_{i}(\tilde{x}, h) .
$$

This gives an integral over the principal stratum having the same form as in the case of smooth manifold. The other components are even-dimensional, and their dimension is at least by 2 less than the dimension of $B$.

\section{An index formula}

In this section we propose a conjecture for the index formula prompted by the Kawasaki index theorem [13], the index theorem for deformation quantization [5] and the $G$-index formula [8]. For the time being we can prove it only in very particular cases, cf. [7]. We hope however to find a complete proof. 
Restricting ourselves to the simplest case of a compact orbifold $B$, we define the index of the algebra of quantum observables $W_{\mathcal{D}}$ as $\operatorname{Tr}_{\text {orb }} 1$.

Let us look at the integral over one of the fixed point orbifolds $F_{m}$ in (7.27), (7.28). Assuming that the original coefficient bundle was $K=\operatorname{End}(E)$ over $B$, one can recognize in this integral an expression for the orbifold trace for a deformation quantization on $F_{m}$ with a coefficient bundle $K \otimes W(N)$ where $W(N)$ is the Weyl algebra in fibers of the normal bundle $N$ of $F_{m}$ with the coefficient trace on $W(N)$ equal to $\operatorname{Tr}_{g}$. Treating $W(N)$ as the isomorphism bundle of the Fock bundle $\mathbb{F}(N)$ and proceeding by analogy with the index theorem for deformation quantization, cf. [5], we come to the following conjecture

$$
\operatorname{Tr}_{\text {orb }} 1=\sum_{k} \frac{1}{m\left(F_{k}\right)} \int_{F_{k}} \exp \left(\frac{\omega_{F_{k}}}{2 \pi h}\right) \operatorname{ch}_{g}(E \otimes \mathbb{F}(N)) \widehat{A}\left(F_{k}\right)
$$

Here $\mathrm{ch}_{g}$ means the character of the bundle $E \otimes \mathbb{F}(N)$ with respect to $\operatorname{Tr}_{g}$. In more detail,

$$
\begin{aligned}
\operatorname{ch}_{g}(E \otimes \mathbb{F}(N)) & =\operatorname{ch}_{g} E \operatorname{ch}_{g} \mathbb{F}(N) \\
& =\operatorname{tr} T(g) \exp \left(\frac{R^{E}}{2 \pi \imath}\right) \operatorname{Tr}_{g} \exp \left(\frac{R^{\mathbb{F}}}{2 \pi \imath}\right)
\end{aligned}
$$

To define the character of the Fock bundle, we introduce an Hermitian connection $\partial^{N}$ on the normal bundle $N$ and an associated connection on $\mathbb{F}(N)$. If

$$
\Gamma^{N}=\Gamma_{\alpha \beta k}^{N} d \tilde{x}^{k}
$$

is the connection form on $N$, then we take the form

$$
\Gamma^{\mathbb{F}}=\frac{1}{2 h} z^{*} \Gamma^{N} * z=\frac{1}{2 h} \Gamma_{\alpha \beta k}^{N} \bar{z}^{\alpha} * z^{\beta} d \tilde{x}^{k}
$$

as the connection form on $\mathbb{F}(N)$. The normal ordering in the last expression is very important, it implies that the vacuum is covariantly constant: $\partial^{N} e_{0}=0$. The curvature of this connection is

$$
R^{\mathbb{F}}=\frac{1}{2 h} z^{*} R^{N} * z=\frac{1}{4 h} R_{\alpha \beta k l}^{N} \bar{z}^{\alpha} * z^{\beta} d \tilde{x}^{k} \wedge d \tilde{x}^{l}
$$

where $R^{N}$ means the curvature of the normal bundle $N$.

Lemma 8.1 The following formula holds

$$
\operatorname{Tr}_{g} \exp \left(\frac{R^{\mathbb{F}}}{2 \pi \imath}\right)=1 / \operatorname{det}\left(1-g^{-1} \exp \left(\frac{R^{N}}{2 \pi \imath}\right)\right) .
$$


Proof. The differential form

$$
S(t)=\exp \left(\frac{t R^{\mathbb{F}}}{2 \pi \imath}\right)
$$

is meaningful as an operator in the Fock space where $h \in(0,1]$ is a number. Indeed, $S$ is a polynomial in $z, \bar{z}$, so the action (7.15) is well-defined. Because of the normal ordering this action is trivial on the vacuum vector, i.e. $S * p=p$. Thus, for a vector $u=a(z, \bar{z}) * p \in \mathbb{F}$ we have

$$
\begin{aligned}
S u & =S * a * p \\
& =S * a * S^{-1} * p .
\end{aligned}
$$

Thus, we need to know the adjoint action of $S$ on $W$, and because it is an automorphism of $W$, it is sufficient to know the action on generators $z^{i}, \bar{z}^{i}$. It may be calculated using a well-known formula

$$
\exp (A) B \exp (-A)=\exp ([A, \cdot]) B
$$

Since $R^{\mathbb{F}}$ is quadratic in generators, we have

$$
\left[\frac{R^{\mathbb{F}}}{2 \pi \imath}, z^{i}\right]=\left[\frac{z^{*} R^{N} * z}{2 h 2 \pi \imath}, z^{i}\right]=-\left(\frac{R^{N} z}{2 \pi \imath}\right)^{i}
$$

and similarly

$$
\left[\frac{R^{\mathbb{F}}}{2 \pi \imath}, \bar{z}^{i}\right]=\left[\frac{z^{*} R^{N} * z}{2 h 2 \pi \imath}, \bar{z}^{i}\right]=\left(\frac{z^{*} R^{N}}{2 \pi \imath}\right)^{i}
$$

If $z$ denotes a column and $z^{*}$ a row, then it results in

$$
\begin{aligned}
S * z * S^{-1} & =\exp \left(-\frac{R^{N}}{2 \pi \imath}\right) z=s^{-1} z, \\
S * z^{*} * S^{-1} & =z^{*} \exp \left(\frac{R^{N}}{2 \pi \imath}\right)=z^{*} s
\end{aligned}
$$

where $s$ denotes the matrix $\exp \left(R^{N} / 2 \pi \imath\right)$. Now, by the definition of the trace $\operatorname{Tr}_{g}$, we get

$$
\begin{aligned}
\operatorname{Tr}_{g} S & =\operatorname{Tr} \hat{g} S \\
& =\sum_{\alpha}\left(\hat{g} S e_{\alpha}, e_{\alpha}\right) \\
& =\int_{\mathbb{C}^{n}} \sum_{\alpha} \frac{p * z^{\alpha} *\left(z^{*} g^{-1} s\right)^{\alpha} * p}{\alpha !(2 h)^{|\alpha|}} \exp \left(\frac{\omega}{2 \pi h}\right) .
\end{aligned}
$$

The series (which in fact is a finite sum) under the integral sign may be simplified as follows. First, the last factor $p$ may be written in the first place, 
because the integral is a trace for the Weyl algebra on $\mathbb{C}^{n}$, and then may be omitted since $p$ is a projector with respect to $*$-product on $W$. Further, for a polynomial $f(z)$ the following formula is true

$$
p * f=\sum_{\alpha} \frac{(-h)^{|\alpha|}}{\alpha !} \frac{\partial^{\alpha} p}{\partial \bar{z}^{\alpha}} f^{(\alpha)}(z)=p \sum_{\alpha} \frac{z^{\alpha}}{\alpha !} f^{(\alpha)}(z)=p f(2 z) .
$$

Finally, using the trace property of the integral, we may omit the last remaining $*$-product, obtaining

$$
\int_{\mathbb{C}^{n}} a * b d z d \bar{z}=\int_{\mathbb{C}^{n}} a b d z d \bar{z}
$$

so that the trace formula reduces to

$$
\begin{aligned}
\operatorname{Tr}_{g} S & =\int_{\mathbb{C}^{n}} p \sum_{k=0}^{\infty} \frac{\left(z^{*} g^{-1} s z\right)^{k}}{k ! h^{k}} \exp \left(\frac{\omega}{2 \pi h}\right) \\
& =\int_{\mathbb{C}^{n}} 2^{n} \exp \left(-\frac{1}{h} z^{*}\left(1-g^{-1} s\right) z\right) \exp \left(\frac{\omega}{2 \pi h}\right) .
\end{aligned}
$$

Calculating this Gaussian integral, we come to (8.2).

Thus, our conjecture takes the form

$$
\operatorname{Tr}_{\text {orb }} 1=\sum_{k} \frac{1}{m\left(F_{k}\right)} \int_{F_{k}} \exp \left(\frac{\omega_{F_{k}}}{2 \pi h}\right) \frac{\operatorname{ch}_{g} E}{\operatorname{det}\left(1-g^{-1} \exp \left(R^{N} / 2 \pi \imath\right)\right)} \widehat{A}\left(F_{k}\right) .
$$

\section{$9 \quad$ Examples}

The purpose of this section is to interpret an index theorem for deformation quantization as an eigenvalue problem. In a particular case we come to our index theorem for symplectic orbifolds.

Consider the symplectic space $M=\mathbb{C}^{2}$ with a standard symplectic form

$$
\omega=\frac{1}{2 \imath}\left(d \bar{z}_{1} \wedge d z_{1}+d \bar{z}_{2} \wedge d z_{2}\right)
$$

and the Hamiltonian action of the group $G=\mathbb{R}$ with the Hamiltonian

$$
H=\frac{1}{2}\left(\left|z_{1}\right|^{2}+c\left|z_{2}\right|^{2}\right)
$$

where $c>0$ is a fixed number. The orbits are as follows

$$
\begin{aligned}
& z_{1} \mapsto e^{-\imath t} z_{1}, \\
& z_{2} \mapsto e^{-\imath c t} z_{2},
\end{aligned}
$$

for $t \in \mathbb{R}$, and we can distinguish three different cases: 
1) $c=1$;

2) $c$ is a rational number, $c \neq 1$ (we take further $c=3$ );

3) $c$ is an irrational number.

Take a number $\lambda$ which is a non-critical value of the Hamiltonian, that is $\lambda>0$, and consider symplectic reduction at the level $\lambda$ in each of the three cases.

For $c=1$ the level set $H=\lambda$ is a sphere $M_{0}=S^{3}$ in $\mathbb{C}^{2}$ and all the orbits are periodic, so, taking $t \in \mathbb{R} \bmod 2 \pi$, we have a free action (9.3) of the group $G=\mathrm{U}(1)=\mathbb{S}^{1}$. The orbits are big circles, and we thus obtain the Hopf fibration

$$
B=M_{0} / G=S^{3} / \mathbb{S}^{1}=\mathbb{C P}^{1}
$$

whose base is a smooth manifold.

If $c=3$ (this is our main example), the level set $M_{0}$ is an ellipsoid

$$
\left|z_{1}\right|^{2}+c\left|z_{2}\right|^{2}=\sqrt{2 \lambda} .
$$

Taking $t \in \mathbb{R} \bmod 2 \pi$ as in the previous case, we again obtain an action (9.3) of the group $G=\mathrm{U}(1)$ on the level manifold. This time, however, the action is only locally free. Indeed, each point of the type $\left(0, z_{2}\right)$ with $\left|z_{2}\right|=\sqrt{2 \lambda} / 3$ is a fixed point of the action (9.3) with $t=0, \pm 2 \pi / 3$. The orbit space $B=M_{0} / G$ is an orbifold, as we shall soon see.

Finally, in the third case we again obtain the ellipsoid $M_{0}(9.5)$ as a level set but this time each orbit (9.3) is dense in the two-dimensional torus, so the orbit space $M_{0} / G$ is not a Hausdorff space at all and the classical reduction makes no sense.

Let us try now to give a quantum interpretation to the reduction procedure. To this end, suppose first that our quantum observables are operators depending on a positive parameter $h$ and acting in some Hilbert space $E$. Algebraically, the reduction procedure goes in two steps. First we extract the subalgebra $A$ of invariant observables, in quantum case the invariance means that $a$ commutes with $H-\lambda$

$$
[H-\lambda, a]=0 .
$$

This implies that each eigenspace of $H-\lambda$ is invariant with respect to any operator $a \in A$. At the second step we consider the restriction $a_{0}$ of the operator $a \in A$ to the zero eigenspace $E_{0}$ of $H-\lambda$. If the eigenspace is nontrivial, the restriction gives an operator $a_{0}$ in $E_{0}$. We obtain thus an algebra $A_{0}=A /(H-\lambda)$ of operators in the eigenspace $E_{0}$ which may be viewed as the reduced algebra of quantum observables. The multiplicity of the zero 
eigenvalue (which is a positive integer number) is given by the trace of the identity operator $1 \in A_{0}$

$$
\operatorname{dim} E_{0}=\operatorname{Tr} 1 \text {. }
$$

So, the eigenvalue problem may be reformulated in an equivalent way: for a fixed $\lambda$ find admissible values of $h \in \Lambda \subset(0,1]$ for which the multiplicity $\operatorname{Tr} 1$ is non-zero and thus belongs to $\mathbb{N}$.

In the deformation quantization framework we can not pose an eigenvalue problem literally, since our algebra of quantum observables $W_{\mathcal{D}}(M)$ is not an operator algebra. Nevertheless we have good substitutes for the corresponding notions allowing us to reformulate the eigenvalue problem, so that it makes sense for deformation quantization. Namely, we replace the algebra $A_{0}$ by the algebra of flat sections $W_{\mathcal{D}}(B)$ on the base manifold (or orbifold) $B$ and the multiplicity $\operatorname{Tr} 1$ by the index of $W_{\mathcal{D}}(B)$. This concerns, of course, the cases 1 ) and 2), the case 3 ) must be treated separately. It is essential that the index is a polynomial $P(1 / h)$ in $1 / h$, thus numerical values of $h$ may be substituted. We propose the following version of the eigenvalue problem in deformation quantization terms:

For a given $\lambda \in \mathbb{R}$ find $h \in(0,1]$ for which the index $P(1 / h)$ takes positive integer values.

Let us start with the simplest case $c=1$. We compare two versions of the eigenvalue problem, namely the traditional eigenvalue problem for the quantum harmonic oscillator with the Weyl symbol

$$
\begin{aligned}
H & =\frac{1}{2}\left(\bar{z}_{1} z_{1}+\bar{z}_{2} z_{2}\right)-\lambda \\
& =\frac{1}{2}\left(\bar{z}_{1} * z_{1}+\bar{z}_{2} * z_{2}\right)+h-\lambda
\end{aligned}
$$

and the above-mentioned deformation quantization version. In the sequel they will be referred to as the traditional and deformation versions. Note, that in the former version we consider $h$ as a number while in the latter one $h$ is a formal parameter.

The traditional spectrum may be found explicitly using the Fock space representation. In fact, the complete set of eigenfunctions are obtained by acting on the vacuum by creation operators

$$
u_{n_{1}, n_{2}}=\bar{z}_{1}^{n_{1}} \bar{z}_{2}^{n_{2}} * p
$$

with the corresponding eigenvalues equal to

$$
h\left(n_{1}+n_{2}+1\right)-\lambda,
$$

where $n_{1} \geq 0, n_{2} \geq 0$ are integer numbers, cf. Fig. 1 . It follows that the Hamiltonian (9.8) has zero eigenvalue if and only if the ratio $m=\lambda / h$ is a 


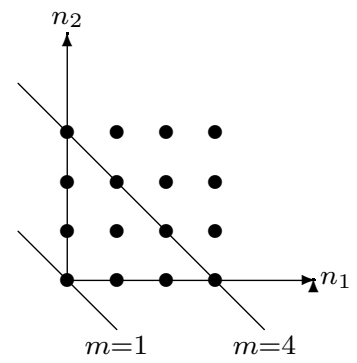

Fig. 1: $\mathrm{c}=1$

positive integer number, this number gives precisely the multiplicity of the eigenvalue.

The deformation version of the eigenvalue problem has the following form. First calculate the index of the reduced algebra $W_{\mathcal{D}}\left(\mathbb{C P}^{1}\right)$ which gives us an a priori multiplicity of the zero eigenvalue of the Hamiltonian (9.8). This begins with the calculation of the reduced symplectic form $\omega_{B}$. Recall that the latter is uniquely defined from the equality

$$
i^{*} \omega_{M}=p^{*} \omega_{B}
$$

on the level manifold $M_{0}$, where $i$ and $p$ are inclusion and projection

$$
M \stackrel{i}{\leftarrow} M_{0} \stackrel{p}{\rightarrow} B
$$

Here $M_{0}$ is the sphere $\left|z_{1}\right|^{2}+\left|z_{2}\right|^{2}=2 \lambda$, and $B$ is the orbit space of the group action

$$
\begin{aligned}
& z_{1} \mapsto e^{-\imath t} z_{1}, \\
& z_{2} \mapsto e^{-\imath t} z_{2},
\end{aligned}
$$

that is the projective space $\mathbb{C P}^{1}$. The orbits may be parametrized by the ratio $\zeta=z_{1} / z_{2}$ if $z_{2} \neq 0$, and by the inverse ratio if $z_{2} \neq 0$. Thus, the symplectic form $\omega_{B}$ should be $\alpha d \bar{\zeta} \wedge d \zeta / 2 \imath$. In polar coordinates

$$
\zeta=\frac{r_{1}}{r_{2}} e^{\imath\left(\varphi_{1}-\varphi_{2}\right)}
$$

so that

$$
\omega_{B}=\frac{\alpha}{2} d\left(\frac{r_{1}^{2}}{r_{2}^{2}}\right) \wedge d\left(\varphi_{1}-\varphi_{2}\right) .
$$

Replacing $r_{2}^{2}=2 \lambda-r_{1}^{2}$ on the sphere $M_{0}$, we find

$$
\omega_{B}=\frac{\alpha \lambda}{\left(2 \lambda-r_{1}^{2}\right)^{2}} d r_{1}^{2} \wedge d\left(\varphi_{1}-\varphi_{2}\right) .
$$


On the other hand, using polar coordinates, we get

$$
\omega_{M}=\frac{1}{2}\left(d r_{1}^{2} \wedge d \varphi_{1}+d r_{2}^{2} \wedge d \varphi_{2}\right)
$$

or, eliminating $r_{2}^{2}$,

$$
\frac{1}{2} d r_{1}^{2} \wedge d\left(\varphi_{1}-\varphi_{2}\right)
$$

Equating these two expressions gives

$$
\begin{aligned}
\alpha & =\frac{\left(2 \lambda-r_{1}^{2}\right)^{2}}{2 \lambda} \\
& =\frac{2 \lambda}{\left(1+|\zeta|^{2}\right)^{2}} .
\end{aligned}
$$

Thus, the reduced form is

$$
\omega_{B}=\frac{\lambda}{\imath} \frac{d \bar{\zeta} \wedge d \zeta}{\left(1+|\zeta|^{2}\right)^{2}}
$$

Integrating this form over $B=\mathbb{C P}^{1}$, we obtain

$$
\operatorname{Tr} 1=\int_{B} \frac{\omega_{B}}{2 \pi h}=\frac{\lambda}{h} .
$$

According to our deformation version of the eigenvalue problem, the spectrum is obtained by equating this ratio to positive integer numbers, the ratio itself being a multiplicity. We see that in this case both spectra, traditional and deformation, coincide.

Consider now the second case $c=3$. The Hamiltonian in this case is

$$
H=\frac{1}{2}\left(\bar{z}_{1} * z_{1}+3 \bar{z}_{2} * z_{2}+4 h\right)-\lambda .
$$

Similarly to the case 1) the traditional spectrum may be calculated explicitly. The eigenfunctions are the same, namely

$$
u_{n_{1}, n_{2}}=\bar{z}_{1}^{n_{1}} \bar{z}_{2}^{n_{2}} * p
$$

with the eigenvalues

$$
h\left(n_{1}+3 n_{2}+2\right)-\lambda,
$$

where $n_{1} \geq 0$ and $n_{2} \geq 0$ are integer numbers. Thus, the Hamiltonian (9.10) has zero eigenvalue if the ratio $\lambda / h$ is equal to $N+2$, for $N=0,1,2, \ldots$, and the multiplicity of this eigenvalue is equal to the number of lattice points on the line $n_{1}+3 n_{2}=N$, cf. Fig. 2. It is convenient to consider three series: $N=3 k, N=3 k+1$ and $N=3 k+2$ where $k=0,1, \ldots$ The multiplicity of 




Fig. 2: $\mathrm{c}=3$

the zero eigenvalue is equal to $k+1$ for each of these series. Let us express the multiplicity as the function of the ratio $\lambda / h$ for each series. If $N=3 k$ then $\lambda / h-2=3 k$, and for the multiplicity $m=k+1$ we obtain

$$
m=\frac{\lambda}{3 h}+\frac{1}{3}
$$

Similarly for series $N=3 k+1$ and $N=3 k+2$, we find

$$
m=\frac{\lambda}{3 h}
$$

and

$$
m=\frac{\lambda}{3 h}-\frac{1}{3}
$$

Thus, we have the following description of the traditional spectrum: $h$ belongs to the spectrum if one of the expressions $\lambda / 3 h-1 / 3, \lambda / 3 h, \lambda / 3 h+1 / 3$ takes positive integer value.

Now, let us calculate the spectrum in deformation version. The zero level set $M_{0}$ is an ellipsoid

$$
\left|z_{1}\right|^{2}+3\left|z_{2}\right|^{2}=2 \lambda
$$

and the action of the group $\mathrm{U}(1)$ is

$$
\begin{aligned}
& z_{1} \mapsto e^{-\imath t} z_{1}, \\
& z_{2} \mapsto e^{-3 \imath t} z_{2} .
\end{aligned}
$$

The orbifold charts for the orbit space are defined by two slices

$$
S_{1}=\left(z_{1}, \sqrt{\frac{2 \lambda-\left|z_{1}\right|^{2}}{3}}\right), \quad\left|z_{1}\right|<\sqrt{2 \lambda},
$$

and

$$
S_{2}=\left(\sqrt{2 \lambda-3\left|z_{2}\right|^{2}}, z_{2}\right), \quad\left|z_{2}\right|<\sqrt{\frac{2 \lambda}{3}} .
$$


The group (9.14) maps $S_{1}$ into itself for $t=0, \pm 2 \pi / 3$, and the only element which maps $S_{2}$ into itself is identity. Thus, for the orbifold charts one can take two discs

$$
\widetilde{O}_{1}=\left\{\left|z_{1}\right|<\sqrt{2 \lambda}\right\}, \quad G_{1}=\mathbb{Z}_{3},
$$

with the standard action of $\mathbb{Z}_{3}$ by multiplication, and

$$
\widetilde{O}_{2}=\left\{\left|z_{2}\right|<\sqrt{2 \lambda / 3}\right\}, \quad G_{2}=1 \text {. }
$$

The Picard group corresponds to the three different characters of the group $G_{1}$, namely,

$$
\chi_{0}(g)=1, \quad \chi_{ \pm 1}(g)=g^{ \pm 1} .
$$

The fixed point orbifolds $F_{k}$ consist of the orbifold $B=M_{0} / \mathrm{U}(1)$ itself corresponding to $g=1$ in each chart, and a zero-dimensional component $F_{0}$ corresponding to non-trivial elements of the group $G_{1}=\mathbb{Z}_{3}$ in the orbifold chart $\widetilde{O}_{1}$, that is $z_{1}=0$.

For a trivial bundle $E$ the term ch $E$ disappears, the term $\widehat{A}(F)$ also disappears since $\operatorname{dim} F \leq 2$. Thus, for each of the three characters our index formula takes the form

$$
\operatorname{ind}_{\chi}=\int_{B} \frac{\omega_{B}}{2 \pi h}+\frac{1}{3} \sum_{g \neq 1} \frac{\chi(g)}{1-g} .
$$

The integral term in the orbifold chart $\widetilde{O}_{1}$ reduces to

$$
\frac{1}{3} \int_{\left|z_{1}\right|<\sqrt{2 \lambda}} \frac{\omega_{B}}{2 \pi h 2 \imath}=\frac{\lambda}{3 h} .
$$

Now, for $\chi_{0}=1$ the additional term in the index formula is equal to

$$
\frac{1}{3}\left(\frac{1}{1-e^{2 \pi \imath / 3}}+\frac{1}{1-e^{-2 \pi \imath / 3}}\right)=\frac{1}{3}
$$

Similarly, for $\chi_{-1}=g^{-1}$

$$
\frac{1}{3}\left(\frac{e^{-2 \pi \imath / 3}}{1-e^{2 \pi \imath / 3}}+\frac{e^{2 \pi \imath / 3}}{1-e^{-2 \pi \imath / 3}}\right)=0,
$$

and for $\chi_{1}=g$

$$
\frac{1}{3}\left(\frac{e^{2 \pi \imath / 3}}{1-e^{2 \pi \imath / 3}}+\frac{e^{-2 \pi \imath / 3}}{1-e^{-2 \pi \imath / 3}}\right)=-\frac{1}{3} .
$$

Thus, we see again that the deformation spectrum coincides with the traditional one. 
We finish by some concluding remarks concerning case 3). The traditional spectrum may be calculated in this case similarly to the previous cases. The result is

$$
h\left(n_{1}+c n_{2}+(c+1) / 2\right)-\lambda .
$$

Since $c$ is irrational, the individual multiplicity of the zero eigenvalue is equal to 1 , if not to zero. Thus, we have no linear function in $\lambda / h$ for the multiplicity as in the previous cases. The situation may be improved if we consider a kind of an averaged multiplicity. For example, let us consider the spectrum in the interval $(-h \varepsilon, h \varepsilon)$, where $\varepsilon$ is a positive number. The number of eigenvalues (9.15) within this interval is approximately equal to

$$
2 \varepsilon\left(\frac{\lambda}{h}+\frac{c+1}{2}\right)
$$

and we may consider the expression in parentheses as a kind of an averaged multiplicity. It is not clear what will play the role of the index in this situation. Hopefully, it will be a kind of a random index [10], we leave this question for further investigations. 
On Index Theorem for Symplectic Orbifolds

\section{References}

[1] M. F. AтזYAh, Elliptic operators and compact groups, Lect. Notes Math. 401, Springer-Verlag, Berlin, 1974.

[2] F. Bayen, M. Flato, C. Fronsdal, A. Lichnerovicz, and D. Sternheimer, Deformation theory and quantization, Ann. Phys. 111 (1978), 61-151.

[3] J. Duistermant, The Heat Kernel Lefschetz Fixed Point Formula for the Spin-c Dirac Operator, Birkhäuser, Boston et al., 1996.

[4] B. Fedosov, A simple geometrical construction of deformation quantization, J. Differential Geom. 40 (1994), 213-238.

[5] B. Fedosov, Deformation Quantization and Index Theory, AkademieVerlag, Berlin, 1995.

[6] B. Fedosov, On normal Darboux coordinates, Amer. Math. Soc. Transl. 206 (2002), no. 2, 81-93.

[7] B. Fedosov, On the trace density in deformation quantization, In: Deformation Quantization. Proceedings of the Meeting of Theoretical Physicist and Mathematicians, Strasbourg, 2001, Walter de Gruyter, Berlin et al., 2002, 67-83.

[8] B. Fedosov, On G-trace and G-index in deformation quantization, Lett. Math. Phys. 52 (2000), 29-49.

[9] B. Fedosov, B.-W. Schulze, and N. Tarkhanov, The index of elliptic operators on manifolds with conical points, Sel. math., New ser. 5 (1999), 1-40.

[10] B. Fedosov, M. Shubin, The index of random elliptic operators, I, II, Mat. Sb. 106 (148) (1978), 108-140; Mat. Sb. 106 (148) (1978), $455-483$.

[11] M. V. Karasev, V. P. Maslov, Asymptotic and geometric quantization, Uspekhi Mat. Nauk 39 (1984), no. 6, 145-173.

[12] T. KaWASAKI, The signature theorem for $V$-manifolds, Topology 17 (1978), 75-83.

[13] T. Kawasaki, The index of elliptic operators over $V$-manifolds, Nagoya Math. J. 84 (1981), 135-157. 
[14] M. Pflaum, On the deformation quantization of symplectic orbispaces, Differential Geometry and its Applications, to appear.

[15] I. Satake, On a generalization of the notion of manifold, Proc. Nat. Acad. Sci. 42 (1956), 359-363.

[16] I. Satake, The Gauss-Bonnet for V-manifolds, J. Math. Soc. Japan 9 (1957), 464-492.

[17] B.-W. Schulze, B. Sternin, and V. Shatalov, On the index of differential operators on manifolds with conical singularities, Annals of Global Anal. and Geom. 16 (1998), 141-172.

[18] M. Vergne, Equivariant index formula for orbifolds, Preprint 94-21, Laboratoire de Mathématiques de l'École Normale Supérieure, Paris, October 1994, 24 pp.

\footnotetext{
E-mail addresses :

fedosov@math.uni-potsdam.de

schulze@math.uni-potsdam.de

tarkhanov@math.uni-potsdam.de
} 